\title{
Stability of Multi-phase Equilibria
}

\author{
Marco Merkli ${ }^{1,2}$
}

1 Department of Mathematics and Statistics, McGill University, 805 Sherbrooke W., Montreal QC, Canada, H3A 2K6 merkli@math.mcgill.ca

2 Centre des Recherches Mathématiques, Université de Montréal, Succursale centre-ville, Montréal, QC, Canada, H3C 3J7

The goal of this note is threefold. I would like to

- explain some recent (1995-) developments in the theory of large quantum systems out of (but close to) equilibrium,

- formulate a notion of "Return to Equilibrium" (dynamical stability) for systems having several equilibria (at fixed temperature), and

- present results on dynamical stability for a specific such system, the "quantum tweezers", a device that can extract particles from a Bose gas with a Bose-Einstein condensate.

\section{Stability of a single-phase equilibrium}

We expect equilibrium states of very large systems to have a property of dynamical stability, called the property of return to equilibrium. This means that if the system is initially in a state that differs only a bit (say locally in space) from an equilibrium state, then it approaches that equilibrium state in the large time limit. One may view this irreversible process as a consequence of the dispersiveness of the dynamics, and the infinite size of the system: the local spatial disturbance of the equilibrium state, defining the initial state, propagates out of any bounded region if one waits long enough. Strictly speaking we expect return to equilibrium only for spatially infinitely extended systems. If one desires to observe localized events of a large, but finite systems (a laboratory) then the above description is a good approximation on an intermediate time scale (time should be large so the system can settle towards the equilibrium state, but not too large as to avoid recurrences in the finite system).

A quantum system for which the property of return to equilibrium is easy to examine is the free Bose gas. If the gas has a Bose-Einstein condensate then the system has many coexisting phases (many equilibrium states at the same temperature). We will explain what the property of return to equilibrium translates into for systems with multiple (multi-phase) equilibria.

If a free Bose gas, modeling an "environment" or a "heat bath", is coupled to a finite system, e.g. to a spin, then it is not so easy any more to show the property of return to equilibrium. This question has been examined, for 
single-phase equilibria, in a variety of recent publications, [14, 15, 3, 6, 17, 10]. The case when the gas is in a condensate state is investigated in [18]. We also refer to [11] for a discussion of the above mentioned validity of the infinite volume approximation of finte systems for intermediate times.

\subsection{The free Bose gas}

Let $\Lambda=[-L / 2, L / 2]^{3} \subset \mathbb{R}^{3}$ be a box in physical space. Pure states of the bose gas localized inside $\Lambda$ are represented by vectors in the bosonic Fock space

$$
\mathcal{F}_{\Lambda}=\bigoplus_{n=0}^{\infty} P_{+} L^{2}\left(\Lambda, \mathrm{d}^{3} x\right)^{\otimes n}
$$

where $P_{+}$projects onto even functions. In order to describe an infinitely extended Bose gas we want to increase the volume $\Lambda \uparrow \mathbb{R}^{3}$. One may replace $\Lambda$ by $\mathbb{R}^{3}$ in expression (1) thereby getting a Hilbert space $\mathcal{F}$ whose vectors represent states (where particles are not constrained to any bounded volume). If the system is in the state $\psi=\left\{\psi_{n}\right\}_{n=0}^{\infty}$ (a normalized vector in $\mathcal{F}$ ) then the probability of finding $n$ particles is given by $p_{n}=\left\|\psi_{n}\right\|_{L^{2}\left(\mathbb{R}^{3 n}, \mathrm{~d}^{3 n} x\right)}^{2}$. Since these probabilities must add up to one we have necessarily that $p_{n} \rightarrow 0$, as $n \rightarrow \infty$. This indicates that if we would like to describe an infinitely extended Bose gas with a fixed (average) density, say one particle per unit volume, then Fock space cannot the right state space.

In order to describe the infinite system we should take the infinite volume limit of the expectation functional ("averages") defined by a vector, or a density matrix on $\mathcal{F}_{\Lambda}$. To this end it is useful to introduce the Weyl algebra $\mathfrak{W}_{\Lambda}=\mathfrak{W}\left(L^{2}\left(\Lambda, \mathrm{d}^{3} x\right)\right)$. This is the $C^{*}$ algebra generated by the unitary Weyl operators,

$$
W(f)=\mathrm{e}^{\mathrm{i} \varphi(f)},
$$

$f \in L^{2}\left(\Lambda, \mathrm{d}^{3} x\right)$, where $\varphi(f)=\frac{1}{\sqrt{2}}\left(a^{*}(f)+a(f)\right)$, and $a^{*}(f), a(f)$ are the usual creation- and annihilation operators on $\mathcal{F}_{\Lambda}$. The Weyl algebra provides us with a very rich class of "observables". Namely, $\mathfrak{W}\left(L^{2}\left(\Lambda, \mathrm{d}^{3} x\right)\right)$ is dense (in the weak operator topology) in $\mathcal{B}\left(\mathcal{F}_{\Lambda}\right)$, the set of all bounded operators on $\mathcal{F}_{\Lambda}$. (This is simply a consequence of the fact that the Weyl algebra acts irreducibly on Fock space.) The Weyl operators satisfy the Canonical Commutation Relations

$$
W(f) W(g)=\mathrm{e}^{-\frac{i}{2} \operatorname{Im}\langle f, g\rangle} W(f+g) .
$$

A state of the system is given by a positive linear functional $\omega_{\Lambda}: \mathfrak{W}_{\Lambda} \rightarrow \mathbb{C}$, normalized as $\omega_{\Lambda}(\mathbb{1 l})=1$. In view of (3) it is not very surprising that any state on the Weyl algebra is entirely determined by its value on the generators of the algebra, i.e., by the (nonlinear) expectation functional

$$
L^{2}\left(\Lambda, \mathrm{d}^{3} x\right) \ni f \mapsto E_{\Lambda}(f):=\omega_{\Lambda}(W(f)) .
$$


The converse is true too: if $E_{\Lambda}: L^{2}\left(\Lambda, \mathrm{d}^{3} x\right) \rightarrow \mathbb{C}$ is a functional satisfying certain compatibility conditions, then it defines uniquely a state $\omega_{\Lambda}$ on $\mathfrak{W}_{\Lambda}$, via $\omega_{\Lambda}(W(f))=E_{\Lambda}(f)$.

The dynamics of Weyl operators is given by

$$
t \mapsto \alpha_{t}^{\Lambda}(W(f))=W\left(\mathrm{e}^{-\mathrm{i} t H_{\Lambda}} f\right),
$$

where the one particle Hamiltonian $H_{\Lambda}$ is $-\Delta$ (Laplace operator acting on $L^{2}\left(\Lambda, \mathrm{d}^{3} x\right)$ with periodic boundary conditions, or a function of this operator).

The procedure of finding (defining!) the infinitely extended system is now reduced to finding the limiting expectation functional $E=\lim _{\Lambda \uparrow \mathbb{R}^{3}} E_{\Lambda}$. We are interested in constructing equilibrium states (w.r.t. the dynamics (5)). Those are special states, characterized by an inverse temperature $\beta$ and a mean density $\bar{\rho}$, which satisfy the so-called KMS condition, [4]. Formally, a state $\omega$ on a $C^{*}$ algebra $\mathfrak{A}$ is a $\left(\beta, \alpha_{t}\right)$-KMS state (where $\alpha_{t}$ is a $*$ automorphism group of $\mathfrak{A}$, and $0<\beta<\infty)$ if the KMS condition is satisfied,

$$
\omega\left(A \alpha_{t}(B)\right)=\omega\left(\alpha_{t-\mathrm{i} \beta}(B) A\right),
$$

for suitable obserbables $A, B \in \mathfrak{A}$.

It is convenient and standard to formulate the theory in Fourier space, where the periodic Laplacian is diagonalized. In the infinite volume limit test functions are elements of $L^{2}\left(\mathbb{R}^{3}, \mathrm{~d}^{3} k\right)$. In the following we place ourselves in this setting (all results can be expressed in direct space at the expense of a more cumbersome notation).

\section{The Araki-Woods construction, [2]}

Let $\mathbb{R}^{3} \ni k \mapsto \rho(k)>0$ be a given function (the "continuous momentumdensity distribution"), and $\rho_{0} \geq 0$ a fixed number (the "condensate density"). Araki and Woods obtain a state of the Bose gas by the following procedure. Put $L^{3} \rho_{0}$ particles in the ground state of the one particle Hamiltonian $H_{\Lambda}$, and a discrete distribution of particles in excited states. Then take the limit $L \rightarrow \infty$ while keeping $\rho_{0}$ fixed and letting the discrete distribution of excited states tend to $\rho(k)$. In this way one obtains the generating functional

$$
E_{\rho, \rho_{0}}^{\mathrm{AW}}(f)=\exp \left[-\frac{1}{4}\left\langle f,\left(1+2(2 \pi)^{3} \rho\right) f\right\rangle\right] J_{0}\left(\sqrt{2(2 \pi)^{3} \rho_{0}}|f(0)|\right),
$$

where $J_{0}\left(\sqrt{\alpha^{2}+\beta^{2}}\right)=\int_{-\pi}^{\pi} \frac{\mathrm{d} \theta}{2 \pi} e^{-\mathrm{i}(\alpha \cos \theta+\beta \sin \theta)}, \alpha, \beta \in \mathbb{R}$ (Bessel function). As we have mentioned above, $E_{\rho, \rho_{0}}^{\mathrm{AW}}$ defines uniquely a state of the infinitely extended Bose gas. The physical interpretation is that the resulting state describes a free Bose gas where a sea of particles, all being in the same state (corresponding to the ground state of the finite-volume Hamiltonian), form a condensate with density $\rho_{0}$, which is immersed in a gas of particles where $\rho(k)$ particles per unit volume have momentum in the infinitesimal volume $\mathrm{d}^{3} k$ 
around $k \in \mathbb{R}^{3}$. Since the Hamiltonian in the finite box is taken with periodic boundary conditions the condensate is homogeneous in space (the ground state wave function is a constant in position space). The resulting state is an equilibrium state (satisfies the KMS condition (6) for $A, B$ Weyl operators with test functions $f \in L^{2}\left(\mathbb{R}^{2}, \mathrm{~d}^{3} k\right)$ and w.r.t. the dynamics $\left.t \mapsto W\left(e^{-i t \omega} f\right)\right)$ if the momentum density distribution is given by

$$
\rho(k)=(2 \pi)^{-3} \frac{1}{\mathrm{e}^{\beta \omega(k)}-1},
$$

corresponding to Planck's law of black body radiation, and the condensate density $\rho_{0} \geq 0$ is arbitrary. The dispersion relation is given by $\omega(k)=|k|^{2}$ or $\omega(k)=|k|$ (non-relativistic Bosons or massless relativistic ones).

We recommend the calculations leading to (7), and the determination of the equilibrium condition on $\rho,(8)$, to the interested reader who wants some hands-on training (a detailed exposition of this, which is of course based on the original article [2], can be found in [19]).

The above procedure yields a generating functional for arbitrary momentum density distributions and the equilibrium situation is a particular case. If we follow the principles of quantum statistical mechanics, the equilibrium state of the infinite system is obtained by taking the thermodynamic limit of local canonical or grand-canonical Gibbs states. This has been done in [5] (canonical case) and in [16] (grand-canonical case). (But see also [2], Section 5.)

\section{The grand-canonical construction, [16]}

The density matrix (acting on Fock space $\mathcal{F}_{\Lambda}$ ) for the local grand-canonical equilibrium system is

$$
\sigma_{\beta, z_{\Lambda}}^{\Lambda}=\frac{\mathrm{e}^{-\beta\left(H_{\Lambda}-\mu_{\Lambda} N_{\Lambda}\right)}}{\operatorname{Tr} \mathrm{e}^{-\beta\left(H_{\Lambda}-\mu_{\Lambda} N_{\Lambda}\right)}},
$$

where $\mu_{\Lambda} \in \mathbb{R}$ is the chemical potential, $z_{\Lambda}=\mathrm{e}^{\beta \mu_{\Lambda}}$ is the fugacity, and $N_{\Lambda}$ is the number operator. For a fixed inverse temperature $0<\beta<\infty$ define the critical density by

$$
\rho_{\text {crit }}(\beta)=(2 \pi)^{-3} \int \frac{\mathrm{d}^{3} k}{\mathrm{e}^{\beta \omega(k)}-1},
$$

and denote by $\bar{\rho} \geq 0$ the total (mean) density of the gas, whose value we are at liberty to choose,

$$
\bar{\rho}=\operatorname{Tr}\left(\sigma_{\beta, z_{\Lambda}}^{\Lambda} \frac{N_{\Lambda}}{L^{3}}\right) .
$$

For each fixed $L$, this determines the value of $z_{\Lambda}$ (as a function of $L, \bar{\rho}$ and $\beta$ ). One then performs the thermodynamic limit of the expectation functional, 


$$
\begin{array}{r}
\text { Stability of Multi-phase Equilibria } \\
E_{\beta, \rho}^{\mathrm{GC}}(f)=\lim _{L \rightarrow \infty} E_{\beta, z_{\Lambda}}^{\Lambda}(f):=\lim _{L \rightarrow \infty} \operatorname{Tr}\left(\sigma_{\beta, z}^{\Lambda} W(f)\right)
\end{array}
$$

where the limit $L \rightarrow \infty$ is taken under the constraint (11). The limiting generating functional (12) is given explicitly by

$$
E_{\beta, \bar{\rho}}^{\mathrm{GC}}(f)= \begin{cases}\mathrm{e}^{-\frac{1}{4}\|f\|^{2}} \exp \left[-\frac{1}{2}\left\langle f, \frac{z_{\infty}}{\mathrm{e}^{\beta \omega}-z_{\infty}} f\right\rangle\right], & \bar{\rho} \leq \rho_{\text {crit }}(\beta) \\ E_{\beta, \bar{\rho}}^{\mathrm{con}}(f), & \bar{\rho} \geq \rho_{\text {crit }}(\beta)\end{cases}
$$

where, with $\rho_{0}:=\bar{\rho}-\rho_{\text {crit }}(\beta) \geq 0$,

$$
E_{\beta, \bar{\rho}}^{\mathrm{con}}(f)=\exp \left[-\frac{1}{4}\left\langle f,\left(1+2(2 \pi)^{3} \rho\right) f\right\rangle\right] \exp \left\{-4 \pi^{3} \rho_{0}|f(0)|^{2}\right\},
$$

and $\rho=\rho(k)$ is given in (8). For subcritical density, $\bar{\rho} \leq \rho_{\text {crit }}(\beta)$, the number $z_{\infty} \in[0,1]$ is determined by the equation

$$
\bar{\rho}=(2 \pi)^{-3} \int \frac{z_{\infty}}{\mathrm{e}^{\beta \omega(k)}-z_{\infty}} \mathrm{d}^{3} k .
$$

In the supercritical case, $\bar{\rho} \geq \rho_{\text {crit }}(\beta)$, we have $z_{\infty}=1$ which corresponds to a vanishing chemical potential, $\mu_{\infty}=0$.

\section{The canonical construction, [5]}

The density matrix of the canonical local Gibbs state is

$$
\mu_{\beta, \bar{\rho}}^{\Lambda}=\frac{\mathrm{e}^{-\beta H_{\Lambda}} P_{\bar{\rho} L^{3}}}{\operatorname{Tr} \mathrm{e}^{-\beta H_{\Lambda}} P_{\bar{\rho} L^{3}}},
$$

and $P_{\bar{\rho}} L^{3}$ is the projection onto the subspace of Fock space with $\bar{\rho} L^{3}$ particles (if $\bar{\rho} L^{3}$ is not an integer take a convex combination of canonical states with integer values $\bar{\rho}_{1} L^{3}$ and $\bar{\rho}_{2} L^{3}$ extrapolating $\bar{\rho} L^{3}$ ). The limiting generating functional is given by

$$
E_{\beta, \bar{\rho}}^{\mathrm{C}}(f)= \begin{cases}\mathrm{e}^{-\frac{1}{4}\|f\|^{2}} \exp \left[-\frac{1}{2}\left\langle f, \frac{z_{\infty}}{e^{\beta \omega}-z_{\infty}} f\right\rangle\right], & \bar{\rho} \leq \rho_{\text {crit }}(\beta) \\ E_{\rho, \rho_{0}}^{\mathrm{AW}}(f), & \bar{\rho} \geq \rho_{\text {crit }}(\beta) .\end{cases}
$$

It coincides with the grand-canonical generating functional in the subcritical case, and with the Araki-Woods generating functional with $\rho$ given by (8) and $\rho_{0}=\bar{\rho}-\rho_{\text {crit }}$ in the supercritical case.

The grand-canonical and the canonical generating functionals are linked, [5], in the supercritical case, $\rho_{0}>0$, by the Laplace transform

$$
E_{\beta, \bar{\rho}}^{\mathrm{con}}(f)=\int_{0}^{\infty} K(r ; \bar{\rho}) E_{\beta, r}^{\mathrm{C}}(f) \mathrm{d} r
$$

where the Kac density $K(r ; \bar{\rho})$ is 


$$
K(r ; \bar{\rho})= \begin{cases}\mathrm{e}^{-\left(r-\rho_{\text {crit }}\right) / \rho_{0}} / \rho_{0}, & r>\rho_{\text {crit }} \\ 0, & r \leq \rho_{\text {crit }}\end{cases}
$$

This means that the grand-canonical equilibrium state with supercritical mean density $\bar{\rho}$ is a superposition of canonical equilibrium states with supercritical densities $r$, weighted with the Kac density $K(r, \bar{\rho})$.

From now on, we focus on the infinite volume equilibrium state $\omega_{\beta, \bar{\rho}}$ determined by the thermodynamic limit of the canonical expectation functional, (17). (All that follows can be carried out for the grand-canonical expectation functional, (13), see [18], but is notationally less cumbersome in the canonical case.)

\subsection{Spontaneous symmetry breaking and multi-phase equilibrium}

We denote by $E_{\rho}^{\mathrm{AW}}(f)$ the Araki-Woods expectation functional (7) at critical density, where $\rho(k)$ is given by $(8)$, and $\rho_{0}=0$. The corresponding equilibrium state is denoted by $\omega_{\beta}$ (it is a state on $\mathfrak{W} \equiv \mathfrak{W}(\mathfrak{D})$, where

$$
\mathfrak{D} \subset L^{2}\left(\mathbb{R}^{3}, \mathrm{~d}^{3} k\right)
$$

consists of functions s.t. the right side of (7) is defined).

With the expansion of the Bessel function $J_{0}$ given after (7) we can write the canonical expectation functional, for $\bar{\rho} \geq \rho_{\text {crit }}(\beta)$, as a superposition

$$
E_{\beta, \bar{\rho}}^{\mathrm{C}}(f)=\int_{S^{1}} \frac{\mathrm{d} \theta}{2 \pi} \mathrm{e}^{-\mathrm{i} \Phi(f, \theta)} E_{\rho}^{\mathrm{AW}}(f),
$$

where the real phase $\Phi$ is given by

$$
\Phi(f, \theta)=(2 \pi)^{-3 / 2} \sqrt{2 \rho_{0}}((\operatorname{Re} f(0)) \cos \theta+(\operatorname{Im} f(0)) \sin \theta) .
$$

Correspondingly, we define the states $\omega_{\beta}^{\theta}$ on $\mathfrak{W} \equiv \mathfrak{W}(\mathfrak{D})$ by

$$
\omega_{\beta}^{\theta}(W(f))=\mathrm{e}^{-\mathrm{i} \Phi(f, \theta)} \omega_{\beta}(W(f)) .
$$

The point of this exercise is to notice that for each $\theta,(23)$ defines a $\beta$-KMS state w.r.t. the dynamics $\alpha^{t}(W(f))=W\left(\mathrm{e}^{-\mathrm{i} t \omega} f\right)$. Thus the supercritical equilibrium state $\omega_{\beta, \rho}$ corresponding to (21) is a uniform superposition of the equilibrium states $\omega_{\beta}^{\theta}, \theta \in S^{1}$. Of course, we can now take any probability measure $\mathrm{d} \mu$ on $S^{1}$ and define a $\left(\beta, \alpha_{t}\right)$-KMS state on $\mathfrak{W}$ by

$$
\omega^{\mu}(\cdot)=\int_{S^{1}} \mathrm{~d} \mu(\theta) \omega_{\beta}^{\theta}(\cdot)
$$

One easily shows that the states (23) are factor states, so they are extremal. We also point out that they are not invariant under the gauge group $\sigma_{s}(W(f))=W\left(\mathrm{e}^{\mathrm{i} s} f\right), s \in \mathbb{R}$, which is a symmetry group of the dynamics 
$\alpha_{t}$ (meaning that $\alpha_{t} \circ \sigma_{s}=\sigma_{s} \circ \alpha_{t}, s, t \in \mathbb{R}$ ). The existence of equilibrium states which have "less symmetry" than the dynamics is called spontaneous symmetry breaking.

We close this paragraph with some observations on space mixing properties. Given a vector $a \in \mathbb{R}^{3}$ we define $\tau_{a}(W(f)):=W\left(f_{a}\right)$, where $f_{a}(x):=$ $f(x-a)$ is the translate of $f$ by $a . \tau_{a}$ defines a (three parameter) group of automorphisms on $\mathfrak{W}$. A state $\omega$ is called strongly mixing w.r.t. space translations if

$$
\lim _{|a| \rightarrow \infty} \omega\left(W(f) \tau_{a}(W(g))\right)=\omega(W(f)) \omega(W(g)),
$$

for any $f, g \in \mathfrak{D}$. This means that if two observables $(W(f)$ and $W(g))$ are spatially separated far from each other then the expectation of the product of the observables is close to the product of the expectation values (independence of random variables). Intuitively, this means that the state $\omega$ has a certain property of locality in space: what happens far out in space does not influence events taking place, say, around the origin. Condition (25) is also called a cluster property. For the equilibrium state $\omega_{\beta, \bar{\rho}}$ corresponding to (17), it is easy to show that

$$
\begin{aligned}
& \lim _{|a| \rightarrow \infty} \omega_{\beta, \bar{\rho}}\left(W(f) \tau_{a}(W(g))\right) \\
& =\omega_{\beta, \bar{\rho}}(W(f)) \omega_{\beta, \bar{\rho}}(W(g)) \begin{cases}1, & \bar{\rho} \leq \rho_{\text {crit }}(\beta) \\
\exp \left[-8 \pi^{3} \rho_{0} \operatorname{Re}(\overline{f(0)} g(0))\right], & \bar{\rho} \geq \rho_{\text {crit }}(\beta) .\end{cases}
\end{aligned}
$$

Consequently, this equilibrium state is strongly mixing w.r.t. space translations if and only if $\rho_{0}=0$, i.e., if and only if there is no condensation. In presence of a condensate, the system exhibits long range correlations (what happens far out does influence what happens say at the origin). On the other hand, it is easily verified that each state $\omega_{\beta}^{\theta}$ is strongly mixing.

\subsection{Return to equilibrium in absence of a condensate}

Consider the equilibrium state $\omega_{\beta, \rho}$ defined by the expectation functional (17), in the regime $\bar{\rho} \leq \rho_{\text {crit }}(\beta)$. We say that $\omega_{\beta, \bar{\rho}}$ has the property of return to equilibrium iff

$$
\lim _{t \rightarrow \infty} \omega_{\beta, \bar{\rho}}\left(B^{*} \alpha_{t}(A) B\right)=\omega_{\beta, \bar{\rho}}\left(B^{*} B\right) \omega_{\beta, \bar{\rho}}(A),
$$

for all $A, B \in \mathfrak{W}$. (This means that all states which are normal w.r.t. $\omega_{\beta, \bar{\rho}}$ converge to $\omega_{\beta, \bar{\rho}}$ in the long time limit.) Let us show that

$$
\lim _{t \rightarrow \infty} \omega_{\beta, \bar{\rho}}\left(W(g) \alpha_{t}(W(f)) W(h)\right)=\omega_{\beta, \bar{\rho}}(W(g) W(h)) \omega_{\beta, \bar{\rho}}(W(f)),
$$

for all $f, g, h \in \mathfrak{D}$. This will clearly imply (26). Using the CCR, (3), we obtain

$$
W(g) W\left(\mathrm{e}^{\mathrm{i} \omega t} f\right) W(h)=\mathrm{e}^{-\frac{\mathrm{i}}{2} \operatorname{Im}\left[\left\langle g, \mathrm{e}^{\mathrm{i} \omega t} f\right\rangle+\left\langle g+\mathrm{e}^{\mathrm{i} \omega t} f, h\right\rangle\right]} W\left(\mathrm{e}^{\mathrm{i} \omega t} f+g+h\right) .
$$


Using the Riemann-Lebesgue lemma, the first factor on the r.h.s. of (28) is seen to have the limit $\mathrm{e}^{-\frac{i}{2} \operatorname{Im}\langle g, h\rangle}$, as $t \rightarrow \infty$. From (17) we obtain

$$
\begin{aligned}
& \omega_{\beta, \bar{\rho}}\left(W\left(\mathrm{e}^{\mathrm{i} \omega t} f+g+h\right)\right) \\
& =\mathrm{e}^{-\frac{1}{4}\left\|\mathrm{e}^{\mathrm{i} \omega t} f+g+h\right\|^{2}} \exp \left[-\frac{1}{2}\left\|\sqrt{\frac{z_{\infty}}{e^{\beta \omega(k)}-z_{\infty}}}\left(\mathrm{e}^{\mathrm{i} \omega t} f+g+h\right)\right\|^{2}\right],
\end{aligned}
$$

and another application of the Riemann-Lebesgue lemma shows that the r.h.s. of (29) tends to $\omega_{\beta, \bar{\rho}}(W(f)) \omega_{\beta, \bar{\rho}}(W(g+h))$, as $t \rightarrow \infty$. That's it as for return to equilibrium for $\bar{\rho} \leq \rho_{\text {crit }}(\beta)$ !

\subsection{Return to equilibrium in presence of a condensate}

Of course one can do the calculation of previous section in the case $\bar{\rho}>\rho_{\text {crit }}$ by using explicitly (17) again. One will then notice that due to the presence of the Bessel function $J_{0}$ in (7), expressions do not split so nicely into products any longer.

A better way of doing things is to realize that the extremal states (23) do have the property of return to equilibrium, as is obvious from the calculation in the previous paragraph and the fact that $\Phi\left(\mathrm{e}^{\mathrm{i} \omega t} f, \theta\right)=\Phi(f, \theta)$. This leads immediately to the following expression for the asymptotic state of an initial condition which is a local perturbation of (normal w.r.t.) a general mixture, (24), of the extremal equilibria:

$$
\lim _{t \rightarrow \infty} \omega^{\mu}\left(B^{*} \alpha_{t}(A) B\right)=\int_{S^{1}} \mathrm{~d} \mu(\theta) \omega_{\beta}^{\theta}\left(B^{*} B\right) \omega_{\beta}^{\theta}(A) .
$$

This shows that the time asymptotic state of the system depends on the state it was initially in. We may interpret this "memory effect" as a consequence of the fact that the system has long-range correlations. Although the perturbation propagates away from any bounded set (the dynamics is dispersive), correlations survive in the limit of large times. If $B$ is such that $\omega_{\beta}^{\theta}\left(B^{*} B\right)=1$ for all $\theta$ in the support of $\mathrm{d} \mu$ (e.g. if $B$ is unitary) then the asymptotic state is just $\omega^{\mu}$ again. In general however, the limit state is a different mixture of the extremal equilibria, with a distribution given by the measure

$$
\mathrm{d} \mu_{B}(\theta)=\omega_{\beta}^{\theta}\left(B^{*} B\right) \mathrm{d} \mu(\theta),
$$

another probability measure, which is absolutely continuous w.r.t. $\mathrm{d} \mu(\theta)$. In particular, the time asymptotic state (30) is normal w.r.t. $\omega^{\mu}$.

\subsection{Spectral approach}

Take a heat reservoir and bring it into contact with a small system, say an $N$-level system (e.g. a spin, or an idealized atom in a cavity). The interaction 
gives rise to emission and absorption processes, where particles in the reservoir (Bosons) are swallowed by the small system, which thereby increases its energy, or where the small system releases a particle by lowering its energy.

It is a well know fact that the coupled system has an equilibrium state (Araki's structural stability of equilibria, see also [7]). We want to show return to equilibrium for the coupled system.

Let us outline here a strategy for doing so, introduced in [14, 15], and further developed in $[3,6,17,9,10,18]$. In Section 3 we apply this method in more detail to the so-called "quantum tweezers", [18].

The remaining part of this section is devoted to presenting the ideas behind a proof of return to equilibrium for a system with a single-phase equilibrium. Certain finer points are neglected in this discussion (c.f. the above references and Section 3).

Assume $\omega$ is a $\left(\beta, \alpha_{t}\right)$-equilibrium state on a $C^{*}$ algebra $\mathfrak{A}$, where $\alpha_{t}$ is a dynamics on $\mathfrak{A}$ (a group of $*$ automorphisms). The GNS construction gives a Hilbert space representation $(\mathcal{H}, \pi, \Omega)$ of the pair $(\mathfrak{A}, \omega)$,

$$
\omega(A)=\langle\Omega, \pi(A) \Omega\rangle,
$$

where $\mathcal{H}$ is the representation Hilbert space, $\Omega \in \mathcal{H}$, and $\pi$ maps $\mathfrak{A}$ into bounded operators on $\mathcal{H}$. There is a unique selfadjoint operator $L$ on $\mathcal{H}$ satisfying

$$
\pi\left(\alpha_{t}(A)\right)=\mathrm{e}^{\mathrm{i} t L} \pi(A) \mathrm{e}^{-\mathrm{i} t L}, \quad L \Omega=0,
$$

for all $A \in \mathfrak{A}, t \in \mathbb{R}$. $L$ is called the (standard) Liouvillian. We have

$$
\begin{aligned}
\omega\left(B^{*} \alpha_{t}(A) B\right) & =\omega\left(\alpha_{-\mathrm{i} \beta}(B) B^{*} \alpha_{t}(A)\right) \\
& =\left\langle\Omega, \pi\left(\alpha_{-\mathrm{i} \beta}(B) B^{*}\right) e^{\mathrm{i} t L} \pi(A) \Omega\right\rangle .
\end{aligned}
$$

We use here that $\omega$ satisfies the KMS condition (6), and (32), (33). It becomes now apparent how we can link the long-time behaviour of (34) to the spectral properties of $L$. If the kernel of $L$ has dimension one (its dimension is at least one, see (33)), and if $L$ has purely absolutely continuous spectrum on $\mathbb{R} \backslash\{0\}$, then the r.h.s. of (34) converges to

$$
\left\langle\Omega, \pi\left(\alpha_{-\mathrm{i} \beta}(B) B^{*}\right) \Omega\right\rangle\langle\Omega, \pi(A) \Omega\rangle=\omega\left(\alpha_{-\mathrm{i} \beta}(B) B^{*}\right) \omega(A),
$$

as $t \rightarrow \infty$. The first term on the r.h.s. is just $\omega\left(B^{*} B\right)$ (use again the KMS condition (6) with $t=0$ ). The combination of (35) and (34) shows that

$$
\lim _{t \rightarrow \infty} \omega\left(B^{*} \alpha_{t}(A) B\right)=\omega\left(B^{*} B\right) \omega(A),
$$

provided $\operatorname{Ker} L=\mathbb{C} \Omega$ and the spectrum of $L$ on $\mathbb{R} \backslash\{0\}$ is purely absolutely continuous. This shows return to equilibrium (c.f. (26))!

A more modest version is return to equilibrium in the ergodic average sense, where the limit in (36) is understood in the ergodic mean sense, 


$$
\lim _{T \rightarrow \infty} \frac{1}{T} \int_{0}^{T} \omega\left(B^{*} \alpha_{t}(A) B\right) \mathrm{d} t=\omega\left(B^{*} B\right) \omega(A) .
$$

Relation (37) follows from (35) and the von Neumann ergodic theorem, provided $\operatorname{Ker} L=\mathbb{C} \Omega$.

\section{Stability of multi-phase equilibria}

The discussion of Section 1.4 motivates the following more abstract

Definitions. 1. Let $\omega$ be a state on a $C^{*}$ algebra $\mathfrak{A}$, invariant w.r.t. a *automorphism group $\alpha_{t}$ of $\mathfrak{A}$. We say that $\omega$ is asymptotically stable (w.r.t. $\left.\alpha_{t}\right)$ if

$$
\lim _{t \rightarrow \infty} \omega\left(B^{*} \alpha_{t}(A) B\right)=\omega\left(B^{*} B\right) \omega(A)
$$

for any $A, B \in \mathfrak{A}$.

2. Let $\omega_{\xi}, \xi \in X$ (a measurable space), be a measurable family of states on a $C^{*}$ algebra $\mathfrak{A}$ (in the sense that $\xi \mapsto \omega_{\xi}(A)$ is measurable for all $A \in \mathfrak{A}$ ) and let $\alpha_{t}$ be a $*$ automorphism group of $\mathfrak{A}$. Given any probability measure $\mu$ on $X$ we define the state

$$
\omega^{\mu}=\int_{X} \mathrm{~d} \mu(\xi) \omega_{\xi}
$$

We say that the family $\omega_{\xi}$ is asymptotically stable (w.r.t. $\alpha_{t}$ ) if, for any $\mu, A, B$, we have

$$
\lim _{t \rightarrow \infty} \omega^{\mu}\left(B^{*} \alpha_{t}(A) B\right)=\int_{X} \mathrm{~d} \mu(\xi) \omega_{\xi}\left(B^{*} B\right) \omega_{\xi}(A) .
$$

3. If $\omega$ in 1 . is a $\left(\beta, \alpha_{t}\right)$-KMS state then we say $\omega$ has the poperty of Return to Equilibrium. Similarly, if the $\omega_{\xi}$ in 2 . are $\left(\beta, \alpha_{t}\right)$-KMS states (then so is $\left.\omega^{\mu}\right)$ we say the family $\omega_{\xi}$ has the property of Return to Equilibrium.

Remarks. 1. More generally one could consider in (39) the case where $\mu$ is a measure on the space of all states on $\mathfrak{A}$. The present setup is sufficient for our purposes.

2. If $\omega_{\xi}$ is asymptotically stable w.r.t. $\alpha_{t}$, for all $\xi$, then the family $\omega_{\xi}$ is asymptotically stable.

In the above definitions the dynamics of the system is given by a (not necessarily norm continuous) $*$ automorphism group $\alpha_{t}$ of a $C^{*}$ algebra $\mathfrak{A}$. While this description applies to free Fermionic or Bosonic heat reservoirs it does not in case a Bosonic reservoir is coupled to a small system. The problem is that one does not know how to define the dynamics for the coupled system as a *automorphism group of the $C^{*}$ algebra of observables (unless the algebra 
is modified, see [9]). One circumvents this issue by defining the interacting dynamics, via a converging perturbation series, as a *automorphism group of the von Neumann algebra associated with a reference state (the uncoupled equilibrium state). We shall therefore adapt the above definitions to a setting where the dynamics is not defined on the level of the $C^{*}$ algebra of observables, but is rather expressed as a ("Schrödinger") dynamics of states.

Definitions. 1'. Let $\omega$ be a state on a $C^{*}$ algebra $\mathfrak{A}$ and denote by $\left(\mathcal{H}_{\omega}, \pi_{\omega}, \Omega_{\omega}\right)$ its GNS representation, $\omega(A)=\left\langle\Omega_{\omega}, \pi_{\omega}(A) \Omega_{\omega}\right\rangle$. Suppose $\sigma_{t}$ is a *automorphism group of the von Neumann algebra $\pi_{\omega}(\mathfrak{A})^{\prime \prime}$. We say that $\omega$ is asymptotically stable (w.r.t. $\sigma_{t}$ ) if

$$
\lim _{t \rightarrow \infty}\left\langle\Omega_{\omega}, \pi_{\omega}\left(B^{*}\right) \sigma_{t}\left(\pi_{\omega}(A)\right) \pi_{\omega}(B) \Omega_{\omega}\right\rangle=\omega\left(B^{*} B\right) \omega(A),
$$

for all $A, B \in \mathfrak{A}$.

2'. Let $\omega_{\xi}, \xi \in X$ (a measurable space), be a measurable family of states on a $C^{*}$ algebra $\mathfrak{A}$ and denote their GNS representations by $\left(\mathcal{H}_{\xi}, \pi_{\xi}, \Omega_{\xi}\right)$. Suppose that, for each $\xi, \sigma_{t}^{\xi}$ is a $*$ automorphism group of the von Neumann algebra $\pi_{\xi}(\mathfrak{A})^{\prime \prime}$, s.t. $\xi \mapsto\left\langle\sigma_{t}^{\xi}(A)\right\rangle_{\pi_{\xi}(B) \Omega_{\xi}}$ is measurable, for all $A, B \in \pi_{\xi}(\mathfrak{A})^{\prime \prime}$, $t \in \mathbb{R} .\left(\langle A\rangle_{\Omega}=\langle\Omega, A \Omega\rangle\right.$.) We say that the family $\omega_{\xi}$ is asymptotically stable (w.r.t. $\sigma_{t}^{\xi}$ ) if, for any $A, B \in \mathfrak{A}$, we have

$$
\lim _{t \rightarrow \infty} \int_{X} \mathrm{~d} \mu(\xi)\left\langle\sigma_{t}^{\xi}\left(\pi_{\xi}(A)\right)\right\rangle_{\pi_{\xi}(B) \Omega_{\xi}}=\int_{X} \mathrm{~d} \mu(\xi) \omega_{\xi}\left(B^{*} B\right) \omega_{\xi}(A),
$$

where $\mu$ is an arbitrary probability measure on $X$.

3'. If $\omega$ in 1'. is a $\left(\beta, \sigma_{t}\right)$-KMS state of $\pi_{\omega}(\mathfrak{A})^{\prime \prime}$ then we say $\omega$ has the poperty of Return to Equilibrium. Similarly, if the $\omega_{\xi}$ in 2'. are $\left(\beta, \sigma_{t}^{\xi}\right)$-KMS states of $\pi_{\xi}(\mathfrak{A})^{\prime \prime}$ we say the family $\omega_{\xi}$ has the property of Return to Equilibrium.

Remark. In case $\sigma_{t}^{\xi}\left(\pi_{\xi}(A)\right)=\pi_{\xi}\left(\alpha_{t}(A)\right)$ for some $*$ automorphism group $\alpha_{t}$ of $\mathfrak{A}$, the second set of definitions reduces to the first one.

In this note we present results of [18], on a (weak) version of relation (42), for equilibrium states of the Bose gas with a condensate interacting with a small system, where the time limit is taken in the ergodic mean sense and is followed by the limit of small coupling constant.

\section{Quantum tweezers}

In the first paragraph we explain the model and the stability result without entering into much technical detail. In Subsections $3.1-3.3$ we give a detailed exposition of the model and results, destined to the more avid reader. 


\section{Description of model and stability result}

The small system with which the Bose gas with condensate interacts can trap finitely many Bosons - we call it therefore a quantum dot. One can imagine the use of such a trap to remove single (uncharged) particles from a reservoir, hence the name quantum tweezers (see also [8]).

The pure states of the small system are given by normalized vectors in $\mathbb{C}^{d}$. We interpret $[1,0, \ldots, 0]$ as the ground state (or "vacuum state"), $[0,1,0, \ldots, 0]$ as the first excited state, e.t.c. The Hamiltonian is given by the diagonal matrix

$$
H_{1}=\operatorname{diag}(0,1,2, \ldots, d-1) .
$$

Our method applies to any selfadjoint diagonal matrix with non-degenerate spectrum. We introduce the raising and lowering operators, $G_{+}$and $G_{-}$,

$$
G_{+}=\left[\begin{array}{cccc}
0 & 0 & \cdots & 0 \\
1 & 0 & \ddots & \vdots \\
\vdots & \ddots & \ddots & 0 \\
0 & \cdots & 1 & 0
\end{array}\right], \quad G_{-}=\left(G_{+}\right)^{*}
$$

$\left(G_{+}\right.$has ones on its subdiagonal) which satisfy $H_{1} G_{ \pm}=G_{ \pm}\left(H_{1} \pm 1\right)$. The action of $G_{+}\left(G_{-}\right)$increases (decreases) the excitation level of the quantum dot by one. The dynamics of an observable $A \in \mathcal{B}\left(\mathbb{C}^{d}\right)$ (bounded operators on $\mathbb{C}^{d}$ ) is given by

$$
\alpha_{1}^{t}(A)=\mathrm{e}^{\mathrm{i} t H_{1}} A \mathrm{e}^{-\mathrm{i} t H_{1}}, \quad t \in \mathbb{R} .
$$

The observable algebra of the combined system is the $C^{*}$-algebra

$$
\mathfrak{A}=\mathcal{B}\left(\mathbb{C}^{d}\right) \otimes \mathfrak{W}(\mathfrak{D}),
$$

where $\mathfrak{D} \subset L^{2}\left(\mathbb{R}^{3}, \mathrm{~d}^{3} k\right)$ (Fourier space) consists of $f \in L^{2}\left(\mathbb{R}^{3},(1+\rho) \mathrm{d}^{3} k\right)$ which are continuous at zero. The non-interacting dynamics is

$$
\alpha_{0}^{t}=\alpha_{1}^{t} \otimes \alpha_{2}^{t},
$$

where

$$
\alpha_{2}^{t}(W(f))=W\left(\mathrm{e}^{\mathrm{i} t \omega} f\right),
$$

$\omega(k)=|k|^{2}$, is the free field dynamics. Denote by $\omega_{1, \beta}$ the Gibbs state of the quantum dot, and let $\omega_{\beta}^{\theta}$ be given as in (23). Then

$$
\omega_{\beta, 0}^{\theta}:=\omega_{1, \beta} \otimes \omega_{\beta}^{\theta}
$$

is a $\left(\beta, \alpha_{0}^{t}\right)$-KMS state. We can form different equilibrium states by mixing such states according to any probability measure $\mu$ on $S^{1}$. Let us now introduce an interaction operator, formally given by the expression 


$$
\lambda\left(G_{+} \otimes a(g)+G_{-} \otimes a^{*}(g)\right),
$$

where $\lambda \in \mathbb{R}$ is a coupling constant, the $G_{ \pm}$are the raising and lowering operators, (44), and $a^{\#}(g)$ are creation $(\#=*)$ and annihilation operators of the heat bath, smeared out with a function $g \in \mathfrak{D}$, called a form factor. The operator $G_{+} \otimes a(g)$ destroys a Boson and traps it in the quantum dot (whose excitation level is thereby increased by one) and similarly, the effect of $G_{-} \otimes a^{*}(g)$ is to release a Boson from the quantum dot. The total number of particles, measured by the "observable" $H_{1}+\int_{\mathbb{R}^{3}} a^{*}(k) a(k) d^{3} k$, is preserved by the interaction (meaning that (50) commutes with this operator). Since the quantum dot can absorb only finitely many Bosons, the interacting equilibrium state is a (local) perturbation of the non-interacting one. A physically different situation occurs when the condensate is coupled to another reservoir. The time-asymptotic states are non-equilibrium stationary states.

As we show in Section 3.2, the system has equilibrium states w.r.t. the interacting dynamics, and there is again a special family among them (extremal factorial ones), $\omega_{\beta, \lambda}^{\theta}$, labelled by $\theta \in S^{1}$, compare with (49).

Let $\mu$ be a probability measure on $S^{1}$ and set

$$
\omega^{\mu}=\int_{S^{1}} \mathrm{~d} \mu(\theta) \omega_{\beta, \lambda}^{\theta} .
$$

Our weak coupling result on Return of Equilibrium, Theorem 1, says that for all $\mu, A, B$

$$
\lim _{\lambda \rightarrow 0} \lim _{T \rightarrow \infty} \frac{1}{T} \int_{0}^{T} \mathrm{~d} t \omega^{\mu}\left(B^{*} \sigma_{\lambda}^{t}(A) B\right)=\int_{S^{1}} \mathrm{~d} \mu(\theta) \omega_{\beta, 0}^{\theta}\left(B^{*} B\right) \omega_{\beta, 0}^{\theta}(A),
$$

where $\sigma_{\lambda}^{t}$ is the interacting dynamics. (The expression $\sigma_{\lambda}^{t}(A)$ has to be understood cum grano salis, in the sense of Definition 2', as $\sigma_{\lambda}^{t}$ can only be defined on the von Neumann algebra of observables, c.f. Section 3.2).

We prove (52) under a condition of regularity and "effectiveness" of the interaction. Let us close this section by discussing the physical meaning of the latter condition. Consider first the Bose gas at critical density $\rho_{\text {crit }}(\beta)$ for some fixed temperature $1 / \beta$ (so that there is no condensate, $\rho_{0}=0$ ). Heuristically, the probability of trapping a Boson in a state $f$ in the quantum dot is given by

$$
\left|\left\langle\left(G_{+} \otimes a(f)\right)(\varphi \otimes \widetilde{\Omega}), \mathrm{e}^{-\mathrm{i} t H_{\lambda}}(\varphi \otimes \widetilde{\Omega})\right\rangle\right|^{2}
$$

where $\varphi$ an eigenstate of the quantum dot Hamiltonian and the Bose gas is in the equilibrium state $\widetilde{\Omega}$ (for the calculation, we put the system in a box and $\widetilde{\Omega}$ is a vector in Fock space with Bosons distributed according to a discrete distribution approaching the Planck distribution as the box size increases). 
The interacting Hamiltonian is $H_{\lambda}=H_{0}+\lambda\left(G_{+} \otimes a(g)+G_{-} \otimes a^{*}(g)\right)$. The second order contribution in $\lambda$ to (53), for large values of $t$, is

$$
P_{2}=C \frac{\lambda^{2}}{\left(\mathrm{e}^{\beta \omega(1)}-1\right)^{2}}|f(1) g(1)|^{2},
$$

where we assume that $f(r), g(r)$ are radially symmetric, and where $C$ is a constant independent of $\beta, f, g . P_{2}$ gives the probability of the second order process where a Boson gets trapped in the quantum dot; the excitation energy is 1 (the quantum dot Hamiltonian (43) has equidistant eigenvalues) and the probability density of finding a Boson with energy $\omega(1)=1$ per unit volume is $\propto\left(\mathrm{e}^{\beta}-1\right)^{-1}$, according to (8). In order not to suppress this trapping process at second order in the coupling constant we assume that $g(1) \neq 0$ ("effective coupling").

Next let us investigate the influence of the condensate. For this we fix a density $\rho_{0}$ of the Bose gas and consider very low temperatures $(\beta \rightarrow \infty)$, so that most particles are in the condensate. If $\widetilde{\Omega}$ denotes the corresponding state of the Bose gas then we calculate the second order in $\lambda$ of (53) to be

$$
Q_{2}(t)=C(1-\cos t) \lambda^{2} \rho_{0}^{2}|f(0) g(0)|^{2} .
$$

We see from (55) that if $g(0)=0$ then there is no coupling to the modes of the condensate: a physically trivial situation where the condensate evolves freely and the small system coupled to the "excited modes" undergoes return to equilibrium. In outline in this note a theory established in [18], which includes the case $g(0) \neq 0$, a situation which could not be handled by approaches developped so far.

\subsection{Non-interacting system}

The states of the small system are determined by density matrices $\rho$ on the finite dimensional Hilbert space $\mathbb{C}^{d}$. A density matrix is a positive trace-class operator, normalized as $\operatorname{Tr} \rho=1$, and the corresponding state

$$
\omega_{\rho}(A)=\operatorname{Tr}(\rho A), \quad A \in \mathcal{B}\left(\mathbb{C}^{d}\right)
$$

is a normalized positive linear functional on the $C^{*}$-algebra $\mathcal{B}\left(\mathbb{C}^{d}\right)$ of all bounded operators on $\mathbb{C}^{d}$, which we call the algebra of observables. The (Heisenberg-) dynamics of the small system is given by (45). Denote the normalized eigenvector of $H_{1}$ corresponding to $E_{j}=j$ by $\varphi_{j}$. Given any inverse temperature $0<\beta<\infty$ the Gibbs state $\omega_{1, \beta}$ is the unique $\beta$-KMS state on $\mathcal{B}\left(\mathbb{C}^{d}\right)$ associated to the dynamics (45). The corresponding density matrix is

$$
\rho_{\beta}=\frac{\mathrm{e}^{-\beta H_{1}}}{\operatorname{Tr} \mathrm{e}^{-\beta H_{1}}} .
$$

Let $\rho$ be a density matrix of rank $d$ (equivalently, $\rho>0$ ) and let $\left\{\varphi_{j}\right\}_{j=0}^{d-1}$ be an orthonormal basis of eigenvectors of $\rho$, corresponding to eigenvalues 
$0<p_{j}<1, \sum_{j} p_{j}=1$. The GNS representation of the pair $\left(\mathcal{B}\left(\mathbb{C}^{d}\right), \omega_{\rho}\right)$ is given by $\left(\mathcal{H}_{1}, \pi_{1}, \Omega_{1}\right)$, where the Hilbert space $\mathcal{H}_{1}$ and the cyclic (and separating) vector $\Omega_{1}$ are

$$
\begin{aligned}
\mathcal{H}_{1} & =\mathbb{C}^{d} \otimes \mathbb{C}^{d}, \\
\Omega_{1} & =\sum_{j} \sqrt{p_{j}} \varphi_{j} \otimes \varphi_{j} \in \mathbb{C}^{d} \otimes \mathbb{C}^{d},
\end{aligned}
$$

and the representation map $\pi_{1}: \mathcal{B}\left(\mathbb{C}^{d}\right) \rightarrow \mathcal{B}\left(\mathcal{H}_{1}\right)$ is

$$
\pi_{1}(A)=A \otimes \mathbb{1}
$$

We introduce the von Neumann algebra

$$
\mathfrak{M}_{1}=\mathcal{B}\left(\mathbb{C}^{d}\right) \otimes \mathbb{1}_{\mathbb{C}^{d}} \subset \mathcal{B}\left(\mathcal{H}_{1}\right) .
$$

The modular conjugation operator $J_{1}$ associated to the pair $\left(\mathfrak{M}_{1}, \Omega_{1}\right)$ is given by

$$
J_{1} \psi_{\ell} \otimes \psi_{r}=\mathcal{C}_{1} \psi_{r} \otimes \mathcal{C}_{1} \psi_{\ell}
$$

where $\mathcal{C}_{1}$ is the antilinear involution $\mathcal{C}_{1} \sum_{j} z_{j} \varphi_{j}=\sum_{j} \overline{z_{j}} \varphi_{j}$ (complex conjugate). According to (59) and (57) the vector $\Omega_{1, \beta}$ representing the Gibbs state $\omega_{1, \beta}$ is given by

$$
\Omega_{1, \beta}=\frac{1}{\sqrt{\operatorname{Tr} \mathrm{e}^{-\beta H_{1}}}} \sum_{j} \mathrm{e}^{-\beta E_{j} / 2} \varphi_{j} \otimes \varphi_{j} \in \mathcal{H}_{1} .
$$

Denote by $\omega_{\rho, \rho_{0}}$ the state on $\mathfrak{W}(\mathfrak{D})$ whose generating functional is $(7)$, where $\rho(k)$ is given in (8), and $\rho_{0} \geq 0$. The GNS representation of the pair $\left(\mathfrak{W}(\mathfrak{D}), \omega_{\rho, \rho_{0}}\right)$ has been given in [2] as the triple $\left(\mathcal{H}_{2}, \pi_{2}, \Omega_{2}\right)$, where the representation Hilbert space is

$$
\mathcal{H}_{2}=\mathcal{F} \otimes \mathcal{F} \otimes L^{2}\left(S^{1}, \mathrm{~d} \sigma\right),
$$

$\mathcal{F}=\mathcal{F}\left(L^{2}\left(\mathbb{R}^{3}, \mathrm{~d}^{3} k\right)\right)$ is the Bosonic Fock space over $L^{2}\left(\mathbb{R}^{3}, \mathrm{~d}^{3} k\right)$ and $L^{2}\left(S^{1}, \mathrm{~d} \sigma\right)$ is the space of $L^{2}$-functions on the circle, with uniform normalized measure $\mathrm{d} \sigma$ $\left(=(2 \pi)^{-1} \mathrm{~d} \theta\right.$, when viewed as the space of periodic functions of $\left.\theta \in[-\pi, \pi]\right)$. The cyclic vector is

$$
\Omega_{2}=\Omega_{\mathcal{F}} \otimes \Omega_{\mathcal{F}} \otimes \mathbf{1}
$$

where $\Omega_{\mathcal{F}}$ is the vacuum in $\mathcal{F}$ and $\mathbf{1}$ is the constant function in $L^{2}\left(S^{1}, \mathrm{~d} \sigma\right)$. The representation map $\pi_{2}: \mathfrak{W}(\mathfrak{D}) \rightarrow \mathcal{B}\left(\mathcal{H}_{2}\right)$ is given by

$$
\pi_{2}(W(f))=W_{\mathcal{F}}(\sqrt{1+\rho} f) \otimes W_{\mathcal{F}}(\sqrt{\rho} \bar{f}) \otimes \mathrm{e}^{-\mathrm{i} \Phi(f, \theta)},
$$

where $W_{\mathcal{F}}(f)=\mathrm{e}^{\mathrm{i} \varphi_{\mathcal{F}}(f)}$ is a Weyl operator in Fock representation and the field operator $\varphi_{\mathcal{F}}(f)$ is 


$$
\varphi_{\mathcal{F}}(f)=\frac{1}{\sqrt{2}}\left(a_{\mathcal{F}}^{*}(f)+a_{\mathcal{F}}(f)\right)
$$

and $a_{\mathcal{F}}^{*}(f), a_{\mathcal{F}}(f)$ are the smeared out creation, annihilation operators satisfying the commutation relations

$$
\left[a_{\mathcal{F}}(f), a_{\mathcal{F}}^{*}(g)\right]=\langle f, g\rangle,\left[a_{\mathcal{F}}(f), a_{\mathcal{F}}(g)\right]=\left[a_{\mathcal{F}}^{*}(f), a_{\mathcal{F}}^{*}(g)\right]=0 .
$$

Our convention is that $f \mapsto a_{\mathcal{F}}(f)$ is an antilinear map. The phase $\Phi \in \mathbb{R}$ is given in (22). In the absence of a condensate $\left(\rho_{0}=0 \Rightarrow \Phi=0\right)$ the third factor in (64)-(66) disappears and the representation reduces to the "Araki-Woods representation" in the form it has appeared in a variety of recent papers. We denote this representation by $\pi_{0}$. More precisely, the GNS representation of $\left(\mathfrak{W}(\mathfrak{D}), \omega_{\rho=0, \rho_{0}=0}\right)$ is given by $\left(\mathcal{F} \otimes \mathcal{F}, \pi_{0}, \Omega_{0}\right)$, where

$$
\begin{aligned}
\pi_{0}(W(f)) & =W_{\mathcal{F}}(\sqrt{1+\rho} f) \otimes W_{\mathcal{F}}(\sqrt{\rho} \bar{f}), \\
\Omega_{0} & =\Omega_{\mathcal{F}} \otimes \Omega_{\mathcal{F}} .
\end{aligned}
$$

Let us introduce the von Neumann algebras

$$
\begin{array}{ll}
\mathfrak{M}_{0}=\pi_{0}(\mathfrak{W}(\mathfrak{D}))^{\prime \prime} & \subset \mathcal{B}(\mathcal{F} \otimes \mathcal{F}) \\
\mathfrak{M}_{2}=\pi_{2}(\mathfrak{W}(\mathfrak{D}))^{\prime \prime} & \subset \mathcal{B}\left(\mathcal{H}_{2}\right)
\end{array}
$$

which are the weak closures (double commutants) of the Weyl algebra represented as operators on the respective Hilbert spaces. $\mathfrak{M}_{2}$ splits into a product

$$
\mathfrak{M}_{2}=\mathfrak{M}_{0} \otimes \mathcal{M} \subset \mathcal{B}(\mathcal{F} \otimes \mathcal{F}) \otimes \mathcal{B}\left(L^{2}\left(S^{1}, \mathrm{~d} \sigma\right)\right),
$$

where $\mathcal{M}$ is the abelian von Neumann algebra of all multiplication operators on $L^{2}\left(S^{1}, \mathrm{~d} \sigma\right)$. It satisfies $\mathcal{M}^{\prime}=\mathcal{M}$. Relation (73) follows from this: clearly we have $\mathfrak{M}_{0}{ }^{\prime} \otimes \mathcal{M} \subset \mathfrak{M}_{2}{ }^{\prime}$, so taking the commutant gives

$$
\mathfrak{M}_{0} \otimes \mathcal{M} \supset \mathfrak{M}_{2}
$$

The reverse inclusion is obtained from $\mathbb{1}_{\mathcal{F} \otimes \mathcal{F}} \otimes \mathcal{M} \subset \mathfrak{M}_{2}$ and $\mathfrak{M}_{0} \otimes \mathbb{1}_{L^{2}\left(S^{1}\right)} \subset$ $\mathfrak{M}_{2}$ (see [2]).

It is well known that $\mathfrak{M}_{0}$, the von Neumann algebra corresponding to the situation without condensate, is a factor. That means that its center is trivial, $\mathfrak{Z}\left(\mathfrak{M}_{0}\right)=\mathfrak{M}_{0} \cap \mathfrak{M}_{0}{ }^{\prime} \cong \mathbb{C}$. However, we have $\mathfrak{Z}\left(\mathfrak{M}_{2}\right)=\left(\mathfrak{M}_{0} \otimes \mathcal{M}\right) \cap\left(\mathfrak{M}_{0}{ }^{\prime} \otimes \mathcal{M}\right)$, i.e.

$$
\mathfrak{Z}\left(\mathfrak{M}_{2}\right)=\mathbb{1}_{\mathcal{F} \otimes \mathcal{F}} \otimes \mathcal{M}
$$

so the von Neumann algebra $\mathfrak{M}_{2}$ is not a factor. One can decompose $\mathfrak{M}_{2}$ into a direct integral of factors, or equivalently, one can decompose $\omega_{\rho, \rho_{0}}$ into an integral over factor states. The Hilbert space (64) is the direct integral

$$
\mathcal{H}_{2}=\int_{[-\pi, \pi]}^{\oplus} \frac{\mathrm{d} \theta}{2 \pi} \mathcal{F} \otimes \mathcal{F}
$$


and the formula (see (65), (66), (69), (70))

$$
\omega_{\rho, \rho_{0}}(W(f))=\left\langle\Omega_{2}, \pi_{2}(W(f)) \Omega_{2}\right\rangle=\int_{-\pi}^{\pi} \frac{\mathrm{d} \theta}{2 \pi} \mathrm{e}^{-\mathrm{i} \Phi(f, \theta)}\left\langle\Omega_{0}, \pi_{0}(W(f)) \Omega_{0}\right\rangle
$$

shows that $\pi_{2}$ is decomposed as

$$
\pi_{2}=\int_{[-\pi, \pi]}^{\oplus} \frac{\mathrm{d} \theta}{2 \pi} \pi_{\theta}
$$

where $\pi_{\theta}: \mathfrak{W}(\mathfrak{D}) \rightarrow \mathcal{B}(\mathcal{F} \otimes \mathcal{F})$ is the representation defined by

$$
\pi_{\theta}(W(f))=\mathrm{e}^{-\mathrm{i} \Phi(f, \theta)} \pi_{0}(W(f)) .
$$

For each fixed $\theta$,

$$
\pi_{\theta}(\mathfrak{W}(\mathfrak{D}))^{\prime \prime}=\mathfrak{M}_{0}
$$

is a factor. Accordingly we have

$$
\mathfrak{M}_{2}=\int_{[-\pi, \pi]}^{\oplus} \frac{\mathrm{d} \theta}{2 \pi} \mathfrak{M}_{0}
$$

Consider the equilibrium state of the uncoupled system

$$
\omega_{\beta, 0}^{\mathrm{con}}=\omega_{1, \beta} \otimes \omega_{2, \beta},
$$

where $\omega_{1, \beta}$ is the Gibbs state of the quantum dot (see (63)), and where $\omega_{2, \beta}$ is the equilibrium state of the heat bath at inverse temperature $\beta$ and above ciritcal density, determined by the generating functional (17), $\bar{\rho} \geq \rho_{\text {crit }}(\beta)$. The index 0 in (82) indicates the absence of an interaction between the two systems. The GNS representation of $\left(\mathfrak{A}, \omega_{\beta, 0}^{\text {con }}\right)$ is just $(\mathcal{H}, \pi, \Omega)$, where

$$
\begin{aligned}
\mathcal{H} & =\mathcal{H}_{1} \otimes \mathcal{H}_{2} \\
\pi & =\pi_{1} \otimes \pi_{2} \\
\Omega_{\beta, 0}^{\text {con }} & =\Omega_{1, \beta} \otimes \Omega_{2} .
\end{aligned}
$$

The free dynamics is $\alpha_{0}^{t},(47)$. Let

$$
\mathfrak{M}_{\beta}^{\text {con }}:=\pi(\mathfrak{A})^{\prime \prime}=\mathfrak{M}_{1} \otimes \mathfrak{M}_{2}=\int_{[-\pi, \pi]}^{\oplus} \frac{\mathrm{d} \theta}{2 \pi} \mathfrak{M}_{1} \otimes \mathfrak{M}_{0} \quad \subset \mathcal{B}(\mathcal{H})
$$

be the von Neumann algebra obtained by taking the weak closure of all observables of the combined system, when represented on $\mathcal{H}$. We have

$$
\pi_{2}\left(\alpha_{2}^{t}(W(f))\right)=\int_{[-\pi, \pi]}^{\oplus} \frac{\mathrm{d} \theta}{2 \pi} \mathrm{e}^{-\mathrm{i} \Phi(f, \theta)} \pi_{0}\left(W\left(\mathrm{e}^{\mathrm{i} \omega t} f\right)\right) .
$$

It is well known and easy to verify that for $A \in \mathfrak{A}$, 


$$
\left(\pi_{1} \otimes \pi_{0}\right)\left(\alpha_{0}^{t}(A)\right)=\mathrm{e}^{\mathrm{i} t L_{0}}\left(\pi_{1} \otimes \pi_{0}\right)(A) \mathrm{e}^{-\mathrm{i} t L_{0}},
$$

where the selfadjoint $L_{0}$ on $\mathcal{H}_{1} \otimes \mathcal{F} \otimes \mathcal{F}$ is given by

$$
\begin{aligned}
& L_{0}=L_{1}+L_{2}, \\
& L_{1}=H_{1} \otimes \mathbb{1}_{\mathbb{C}^{d}}-\mathbb{1}_{\mathbb{C}^{d}} \otimes H_{1}, \\
& L_{2}=\mathrm{d} \Gamma(\omega) \otimes \mathbb{1}_{\mathcal{F}}-\mathbb{1}_{\mathcal{F}} \otimes \mathrm{d} \Gamma(\omega) .
\end{aligned}
$$

Here $\mathrm{d} \Gamma(\omega)$ is the second quantization of the operator of multiplication by $\omega$ on $L^{2}\left(\mathbb{R}^{3}, \mathrm{~d}^{3} k\right)$. We will omit trivial factors 11 or indices $\mathbb{C}^{d}, \mathcal{F}$ whenever we have the reasonable hope that no confusion can arise (e.g. $L_{1}$ really means $\left.L_{1} \otimes \mathbb{1}_{\mathcal{F}} \otimes \mathbb{1}_{\mathcal{F}}\right)$. It follows from (85)-(90) that the uncoupled dynamics $\alpha_{0}^{t}$ is unitarily implemented in $\mathcal{H}$ by

$$
\pi\left(\alpha_{0}^{t}(A)\right)=\mathrm{e}^{\mathrm{i} t \mathcal{L}_{0}} \pi(A) \mathrm{e}^{-\mathrm{i} t \mathcal{L}_{0}},
$$

where the standard, non-interacting Liouvillian $\mathcal{L}_{0}$ is the selfadjoint operator on $\mathcal{H}$ with constant ( $\theta$-independent) fiber $L_{0}$,

$$
\mathcal{L}_{0}=\int_{[-\pi, \pi]}^{\oplus} \frac{\mathrm{d} \theta}{2 \pi} L_{0} .
$$

The r.h.s. of (91) extends to a *automorphism group $\sigma_{0}^{t}$ of $\mathfrak{M}_{\beta}^{\text {con }}$ which is reduced by the decomposition (85). We write

$$
\sigma_{0}^{t}=\int_{[-\pi, \pi]}^{\oplus} \frac{\mathrm{d} \theta}{2 \pi} \sigma_{0, \theta}^{t},
$$

where $\sigma_{0, \theta}^{t}$ is the $*$ automorphism group of $\mathfrak{M}_{1} \otimes \mathfrak{M}_{0}$ generated by $L_{0}$. As is well known,

$$
\Omega_{\beta, 0}=\Omega_{1, \beta} \otimes \Omega_{0}
$$

is a $\left(\beta, \sigma_{0, \theta}^{t}\right)$-KMS state of $\mathfrak{M}_{1} \otimes \mathfrak{M}_{0}$. The modular conjugation operator $J$ associated to $\left(\mathfrak{M}_{0}, \Omega_{1, \beta} \otimes \Omega_{0}\right)$ is

$$
J=J_{1} \otimes J_{0},
$$

where $J_{1}$ is given by $(62)$ and where the action of $J_{0}$ on $\mathcal{F} \otimes \mathcal{F}$ is determined by antilinearly extending the relation

$$
J_{0} \pi_{0}(W(f)) \Omega_{0}=W_{\mathcal{F}}(\sqrt{\rho} f) \otimes W_{\mathcal{F}}(\sqrt{1+\rho} \bar{f}) \Omega_{0} .
$$

$J_{0}$ defines an antilinear representation of the Weyl algebra according to $W(f) \mapsto J_{0} \pi_{0}(W(f)) J_{0}$, which commutes with the representation $\pi_{0}$ given in (69). We view this as a consequence of the Tomita-Takesaki theory which asserts that $\mathfrak{M}_{0}{ }^{\prime}=J_{0} \mathfrak{M}_{0} J_{0}$.

It follows from (84), (85), (93) that 


$$
\Omega_{\beta, 0}^{\text {con }}=\int_{[-\pi, \pi]}^{\oplus} \frac{\mathrm{d} \theta}{2 \pi} \Omega_{\beta, 0}
$$

is a $\left(\beta, \sigma_{0}^{t}\right)$-KMS state on $\mathfrak{M}_{\beta}^{\text {con }}$, and that the modular conjugation operator $\mathcal{J}$ associated to $\left(\mathfrak{M}_{\beta}^{\text {con }}, \Omega_{\beta, 0}^{\text {con }}\right)$ is given by

$$
\mathcal{J}=\int_{[-\pi, \pi]}^{\oplus} \frac{\mathrm{d} \theta}{2 \pi} J_{1} \otimes J_{0} .
$$

The standard Liouvillian $\mathcal{L}_{0},(92)$, satisfies the relation

$$
\mathcal{J} \mathcal{L}_{0}=-\mathcal{L}_{0} \mathcal{J}
$$

One can choose different generators to implement the dynamics $\alpha_{0}^{t}$ on $\mathcal{H}$ (by adding to the standard $\mathcal{L}_{0}$ any selfadjoint element affiliated with the commutant $\left.\left(\mathfrak{M}_{\beta}^{\text {con }}\right)^{\prime}\right)$. The choice $(92)$ is compatible with the symmetry $\mathfrak{M}_{\beta}^{\text {con }} \cong\left(\mathfrak{M}_{\beta}^{\text {con }}\right)^{\prime}$, in that it also implements $\alpha_{0}^{t}$ for the antilinear representation $\mathcal{J} \pi(\cdot) \mathcal{J}$. Another way of saying this is that the standard Liouvillian (92) is the only generator which implements the non-interacting dynamics $\alpha_{0}^{t}$ and satisfies

$$
\mathcal{L}_{0} \Omega_{\beta, 0}^{\text {con }}=0
$$

see e.g. $[4,7]$.

\subsection{Interacting system}

We define the coupled dynamics, i.e. the interaction between the small system and the Bose gas, by specifying a *automorphism group $\sigma_{\lambda}^{t}$ of the von Neumann algebra $\mathfrak{M}_{\beta}^{\text {con }}$ (the "perturbed" or "interacting dynamics"). One may argue that a conceptually more satisfying way is to introduce a representation independent regularized dynamics as a *automorphism group of $\mathfrak{A}$ and then removing the regularization once the dynamics is represented on a Hilbert space. This procedure can be implemented by following the arguments of [9], where it has been carried out for the Bose gas without condensate. The resulting dynamics is of course the same for both approaches. For a technically more detailed exposition of the following construction we refer the reader to $[9]$.

The interaction between the two subsystems is given formally by (50), which we understand as an operator in a regular representation of the Weyl algebra, so that the creation and annihilation operators are well defined.

The field operator $\varphi(f)=\left.\frac{1}{\mathrm{i}} \partial_{t}\right|_{t=0} \pi(W(t f))$ in the representation $\pi,(83)$, is easily calculated to be

$$
\begin{aligned}
\varphi(f) & =\int_{[-\pi, \pi]}^{\oplus} \frac{\mathrm{d} \theta}{2 \pi} \varphi_{\theta}(f), \\
\varphi_{\theta}(f) & =\varphi_{\mathcal{F}}(\sqrt{1+\rho} f) \otimes \mathbb{1}+\mathbb{1} \otimes \varphi_{\mathcal{F}}(\sqrt{\rho} \bar{f})-\Phi(f, \theta),
\end{aligned}
$$


where $\Phi(f, \theta)$ is given in (22), and where $\varphi_{\mathcal{F}}(f)$ is given in (67). The creation operator $a_{\theta}^{*}(f):=\frac{1}{\sqrt{2}}\left(\varphi_{\theta}(f)-\mathrm{i} \varphi_{\theta}(\mathrm{i} f)\right)$ has the expression

$$
a_{\theta}^{*}(f)=a_{\mathcal{F}}^{*}(\sqrt{1+\rho} f) \otimes \mathbb{1}_{\mathcal{F}}+\mathbb{1}_{\mathcal{F}} \otimes a_{\mathcal{F}}(\sqrt{\rho} \bar{f})-(2 \pi)^{-3 / 2} \sqrt{\rho_{0}} f(0) \mathrm{e}^{-\mathrm{i} \theta} .
$$

Define the interaction operator

$$
\begin{aligned}
& V= \int_{[-\pi, \pi]}^{\oplus} \frac{\mathrm{d} \theta}{2 \pi} V_{\theta}, \\
& V_{\theta}=G_{+} \otimes \mathbb{1}_{\mathbb{C}^{d}} \otimes\left(a_{\mathcal{F}}(\sqrt{1+\rho} g) \otimes \mathbb{1}_{\mathcal{F}}+\mathbb{1}_{\mathcal{F}} \otimes a_{\mathcal{F}}^{*}(\sqrt{\rho} \bar{g})\right. \\
&\left.\quad-(2 \pi)^{-3 / 2} \sqrt{\rho_{0}} \overline{g(0)} \mathrm{e}^{\mathrm{i} \theta}\right)+ \text { adjoint },
\end{aligned}
$$

which corresponds formally to $\pi\left(G_{+} \otimes a(g)\right)+$ adjoint (apply (83) to (50)). $V$ is an unbounded selfadjoint operator on $\mathcal{H}$ which is affiliated with $\mathfrak{M}_{\beta}^{\text {con }}$. For $t \in \mathbb{R}, A \in \mathfrak{M}_{\beta}^{\text {con }}$ we set

$$
\begin{gathered}
\sigma_{\lambda}^{t}(A)=\sum_{n \geq 0}(\mathrm{i} \lambda)^{n} \int_{0}^{t} \mathrm{~d} t_{1} \ldots \int_{0}^{t_{n-1}} \mathrm{~d} t_{n}\left[\mathrm{e}^{\mathrm{i} t_{n} \mathcal{L}_{0}} V \mathrm{e}^{-\mathrm{i} t_{n} \mathcal{L}_{0}},[\cdots\right. \\
\left.\left.\cdots\left[\mathrm{e}^{\mathrm{i} t_{1} \mathcal{L}_{0}} V \mathrm{e}^{-\mathrm{i} t_{1} \mathcal{L}_{0}}, A\right] \cdots\right]\right] .
\end{gathered}
$$

The series is understood in the strong sense on a dense set of vectors on which it converges for any $A \in \mathfrak{M}_{\beta}^{\text {con }}, \lambda, t \in \mathbb{R}$. Since $V$ is affiliated with $\mathfrak{M}_{\beta}^{\text {con }}$ and $\mathrm{e}^{\mathrm{i} t \mathcal{L}_{0}}(\cdot) \mathrm{e}^{-\mathrm{i} t \mathcal{L}_{0}}$ leaves $\mathfrak{M}_{\beta}^{\text {con }}$ invariant, one sees that the integrand in (105) does not change when one adds to each $\mathrm{e}^{\mathrm{i} t_{j} \mathcal{L}_{0}} V \mathrm{e}^{-\mathrm{i} t_{j} \mathcal{L}_{0}}$ a term $-\mathcal{J} \mathrm{e}^{\mathrm{i} t_{j} \mathcal{L}_{0}} V \mathrm{e}^{-\mathrm{i} t_{j} \mathcal{L}_{0}} \mathcal{J}=-\mathrm{e}^{\mathrm{i} t_{j} \mathcal{L}_{0}} \mathcal{J} V \mathcal{J} \mathrm{e}^{-\mathrm{i} t_{j} \mathcal{L}_{0}}$ (which is affiliated with the commutant $\left.\left(\mathfrak{M}_{\beta}^{\text {con }}\right)^{\prime}\right)$. In other words, $V$ in (105) can be replaced by $V-\mathcal{J} V \mathcal{J}$. The r.h.s. of (105) is then identified as the Dyson series expansion of

$$
\mathrm{e}^{\mathrm{i} t \mathcal{L}_{\lambda}} A \mathrm{e}^{-\mathrm{i} t \mathcal{L}_{\lambda}},
$$

where the standard, interacting Liouvillian $\mathcal{L}_{\lambda}$ is the selfadjoint operator

$$
\mathcal{L}_{\lambda}=\mathcal{L}_{0}+\lambda(V-\mathcal{J} V \mathcal{J}) \equiv \mathcal{L}_{0}+\lambda \mathcal{I} .
$$

Subtracting the term $\mathcal{J} V \mathcal{J}$ serves to preserve the symmetry (99) under the perturbation, i.e., we have $\mathcal{J} \mathcal{L}_{\lambda}=-\mathcal{L}_{\lambda} \mathcal{J}$. It is not hard to verify that (106) defines a $*$ automorphism group

$$
\sigma_{\lambda}^{t}(A)=\mathrm{e}^{\mathrm{i} t \mathcal{L}_{\lambda}} A \mathrm{e}^{-\mathrm{i} t \mathcal{L}_{\lambda}}
$$

of $\mathfrak{M}_{\beta}^{\text {con }}$, [9]. This defines the interacting dynamics. The Liouvillian $\mathcal{L}_{\lambda}$ is reduced by the direct integral decomposition,

$$
\mathcal{L}_{\lambda}=\int_{[-\pi, \pi]}^{\oplus} \frac{\mathrm{d} \theta}{2 \pi} L_{\lambda, \theta}
$$


where the selfadjoint operator $L_{\lambda, \theta}$ is

$$
L_{\lambda, \theta}=L_{0}+\lambda I_{\theta} .
$$

Here $L_{0}$ is given in (88) and we define

$$
\begin{aligned}
I_{\theta}= & I+K_{\theta} \\
I= & G_{+} \otimes \mathbb{1}_{\mathbb{C}^{d}} \otimes\left\{a_{\mathcal{F}}(\sqrt{1+\rho} g) \otimes \mathbb{1}_{\mathcal{F}}+\mathbb{1}_{\mathcal{F}} \otimes a_{\mathcal{F}}^{*}(\sqrt{\rho} \bar{g})\right\}+\operatorname{adj} \\
& -\mathbb{1}_{\mathbb{C}^{d}} \otimes \mathcal{C}_{1} G_{+} \mathcal{C}_{1} \otimes\left\{a_{\mathcal{F}}^{*}(\sqrt{\rho} g) \otimes \mathbb{1}_{\mathcal{F}}+\mathbb{1}_{\mathcal{F}} \otimes a_{\mathcal{F}}(\sqrt{1+\rho} \bar{g})\right\}+\operatorname{adj} . \\
K_{\theta}= & K_{\theta}^{1} \otimes \mathbb{1}_{\mathbb{C}^{d}} \otimes \mathbb{1}_{\mathcal{F} \otimes \mathcal{F}}-\mathbb{1}_{\mathbb{C}^{d}} \otimes \mathcal{C}_{1} K_{\theta}^{1} \mathcal{C}_{1} \otimes \mathbb{1}_{\mathcal{F} \otimes \mathcal{F}} \\
K_{\theta}^{1}= & -(2 \pi)^{-3 / 2} \sqrt{\rho_{0}}\left(G_{+} \overline{g(0)} \mathrm{e}^{\mathrm{i} \theta}+G_{-} g(0) \mathrm{e}^{-\mathrm{i} \theta}\right)
\end{aligned}
$$

with $\mathcal{C}_{1}, \Phi$ defined in (62), (22) and where the creation and annihilation operators $a_{\mathcal{F}}^{*}, a_{\mathcal{F}}$ are defined by (68). It is convenient to write (compare with (93))

$$
\sigma_{\lambda}^{t}=\int_{[-\pi, \pi]}^{\oplus} \frac{\mathrm{d} \theta}{2 \pi} \sigma_{\lambda, \theta}^{t},
$$

where $\sigma_{\lambda, \theta}^{t}$ is the *automorphism group of $\mathfrak{M}_{1} \otimes \mathfrak{M}_{0}$ generated by $L_{\lambda, \theta},(110)$.

To the interacting dynamics (108) corresponds a $\beta$-KMS state on $\mathfrak{M}_{\beta}^{\text {con }}$, the equilibrium state of the interacting system. It is given by the vector

$$
\Omega_{\beta, \lambda}^{\text {con }}=\left(Z_{\beta, \lambda}^{\text {con }}\right)^{-1} \int_{[-\pi, \pi]}^{\oplus} \frac{\mathrm{d} \theta}{2 \pi} \Omega_{\beta, \lambda}^{\theta},
$$

where $Z_{\beta, \lambda}^{\text {con }}$ is a normalization factor ensuring that $\left\|\Omega_{\beta, \lambda}^{\text {con }}\right\|=1$, and where

$$
\Omega_{\beta, \lambda}^{\theta}=\left(Z_{\beta, \lambda}^{\theta}\right)^{-1} \mathrm{e}^{-\beta\left(L_{0}+\lambda I_{\theta, \ell}\right) / 2} \Omega_{\beta, 0} \in \mathcal{H}_{1} \otimes \mathcal{F} \otimes \mathcal{F} .
$$

$Z_{\beta, \lambda}^{\theta}$ is again a normalization factor, and $I_{\theta, \ell}$ is obtained by dropping the terms coming with a minus sign in the r.h.s. of both (112) and (113). The fact that $\Omega_{\beta, 0},(94)$, is in the domain of the unbounded operator $\mathrm{e}^{-\beta\left(L_{0}+\lambda I_{\theta, \ell}\right) / 2}$, provided

$$
\|g / \sqrt{\omega}\|_{L^{2}\left(\mathbb{R}^{3}\right)}<\infty
$$

can be seen by expanding the exponential in a Dyson series and verifying that the series applied to $\Omega_{\beta, 0}$ converges, see e.g. [3]. It then follows from the generalization of Araki's perturbation theory of KMS states, given in [7], that $\Omega_{\beta, \lambda}^{\theta}$ is a $\left(\beta, \sigma_{\lambda, \theta}^{t}\right)$-KMS state on $\mathfrak{M}_{1} \otimes \mathfrak{M}_{0}$, denoted by

$$
\omega_{\beta, \lambda}^{\theta}(\cdot)=\left\langle\Omega_{\beta, \lambda}^{\theta}, \cdot \Omega_{\beta, \lambda}^{\theta}\right\rangle
$$

and that

$$
L_{\lambda, \theta} \Omega_{\beta, \lambda}^{\theta}=0 .
$$

We conclude that $\Omega_{\beta, \lambda}^{\text {con }}$ is a $\left(\beta, \sigma_{\lambda}^{t}\right)$-KMS state on $\mathfrak{M}_{\beta}^{\text {con }}$, and that $\mathcal{L}_{\lambda} \Omega_{\beta, \lambda}^{\text {con }}=0$. 


\subsection{Stability of the quantum tweezers, main results}

We make two assumptions on the form factor $g$ determining the interaction (see (50), (104)).

(A1) Regularity. The form factor $g$ is a function in $C^{4}\left(\mathbb{R}^{3}\right)$ and satisfies

$$
\left\|(1+1 / \sqrt{\omega})\left(k \cdot \nabla_{k}\right)^{j} \sqrt{1+\rho} g\right\|_{L^{2}\left(\mathbb{R}^{3}, \mathrm{~d}^{3} k\right)}<\infty,
$$

for $j=0, \ldots, 4$, and $\left\|(1+\omega)^{2} \sqrt{1+\rho} g\right\|_{L^{2}\left(\mathbb{R}^{3}, \mathrm{~d}^{3} k\right)}<\infty$.

(A2) Effective coupling. We have $\int_{S^{2}} \mathrm{~d} \sigma|g(1, \sigma)|^{2} \neq 0$, where $g$ is represented in spherical coordinates.

Remarks. 1) Condition (A1) is used in the application of the virial theorem - we choose the generator of dilations $\frac{1}{2}\left(k \cdot \nabla_{k}+\nabla_{k} \cdot k\right)$ to be the conjugate operator in the theory. It is important to notice that in order to couple the particles of the Bose-Einstein condensate to the quantum dot, we must have $g(0) \neq 0$. If the dispersion relation is given by $\omega(k) \sim|k|^{s}$, as $|k| \sim 0$, then (120) is satisfied for $s<3 / 2$. (This does not include non-relativistic Bosons, for which $s=2$.)

2) Condition (A2) is often called the Fermi Golden Rule Condition. It guarantees that the processes of absorption and emission of field quanta by the small system, which are the origin of the stability of the equilibrium, are effective (see the discussion at the beginning of Section 3). We integrate over a sphere of radius one since the gap between neighbouring eigenvalues of $H_{1}$ is equal to one (Bohr frequency).

Let $B \in \mathfrak{A}, \mu$ a probability measure on $S^{1}$, and define a state on $\mathfrak{A}$ by

$$
\omega_{B}^{\mu}(A)=\int_{S^{1}} \mathrm{~d} \mu(\theta) \omega_{\beta, \lambda}^{\theta}\left(B^{*} A B\right),
$$

where $\omega_{\beta, \lambda}^{\theta}(A)=\left\langle\Omega_{\beta, \lambda}^{\theta}, A_{\theta} \Omega_{\beta, \lambda}^{\theta}\right\rangle$, with $A_{\theta}:=\left(\pi_{1} \otimes \pi_{\theta}\right)(A)$ (see (118), (60), (79)). We introduce the suggestive notation

$$
\omega_{B}^{\mu}\left(\sigma_{\lambda}^{t}(A)\right):=\int_{S^{1}} \mathrm{~d} \mu(\theta)\left\langle\sigma_{\lambda, \theta}^{t}\left(A_{\theta}\right)\right\rangle_{B_{\theta} \Omega_{\beta, \lambda}^{\theta}},
$$

where $\langle A\rangle_{\psi}=\langle\psi, A \psi\rangle$, and where $\sigma_{\lambda, \theta}^{t}$ is given in (114).

Theorem 1 (Stability of equilibrium with condensate, [18]). Assume conditions (A1) and (A2). Let $A, B \in \mathfrak{A}$, and let $\mu$ be a probability measure on $S^{1}$. Then

$$
\lim _{\lambda \rightarrow 0} \lim _{T \rightarrow \infty} \frac{1}{T} \int_{0}^{T} \omega_{B}^{\mu}\left(\sigma_{\lambda}^{t}(A)\right) \mathrm{d} t=\int_{S^{1}} \mathrm{~d} \mu(\theta) \omega_{\beta, 0}^{\theta}\left(B^{*} B\right) \omega_{\beta, 0}^{\theta}(A),
$$

where $\omega_{\beta, 0}^{\theta}$ is given in (49). 
Remark. We expect that the stronger result

$$
\lim _{T \rightarrow \infty} \frac{1}{T} \int_{0}^{T} \omega_{B}^{\mu}\left(\sigma_{\lambda}^{t}(A)\right) \mathrm{d} t=\int_{S^{1}} \mathrm{~d} \mu(\theta) \omega_{\beta, \lambda}^{\theta}\left(B^{*} B\right) \omega_{\beta, \lambda}^{\theta}(A)
$$

is true, at least for small values of $\lambda$. This Return to Equilibrium has been proven for systems without a condensate (with varying conditions on the interaction and varying modes of convergence) in several recent works, [14, $15,3,17,6,10]$. The obstruction to applying the strategies of these papers is that they all need the condition that either $g(0)=0$, or $g(k) \sim|k|^{-1 / 2}$, as $|k| \rightarrow 0$. The first case is uninteresting in the presence of a condensate (no coupling to the modes of the condensate!), and the second type of form factor does not enter into the description of a system with a condensate (see $(20))$.

In order to state the virial theorem and to measure regularity of eigenvectors of $L_{\lambda, \theta},(110)$, we introduce the non-negative selfadjoint operator

$$
\Lambda=\mathrm{d} \Gamma(\omega) \otimes \mathbb{1}_{\mathcal{F}}+\mathbb{1}_{\mathcal{F}} \otimes \mathrm{d} \Gamma(\omega),
$$

where $\mathrm{d} \Gamma(\omega)$ is the second quantization of the operator of multiplication by $\omega(k)=|k|^{2}$ on $L^{2}\left(\mathbb{R}^{3}, \mathrm{~d}^{3} k\right)$. The kernel of $\Lambda$ is spanned by the vector $\Omega_{0}=\Omega_{\mathcal{F}} \otimes \Omega_{\mathcal{F}}$ (c.f. (70)) and $\Lambda$ has no nonzero eigenvalues. The operator $\Lambda$ represents the quadratic form i $\left[L_{0}, A\right]$, the commutator of $L_{0}$ with the conjugate operator

$$
A=\mathrm{d} \Gamma\left(a_{\mathrm{d}}\right) \otimes \mathbb{1}_{\mathcal{F}}-\mathbb{1}_{\mathcal{F}} \otimes \mathrm{d} \Gamma\left(a_{\mathrm{d}}\right),
$$

where $a_{\mathrm{d}}$ is the selfadjoint generator of dilations on $L^{2}\left(\mathbb{R}^{3}, \mathrm{~d}^{3} k\right)$,

$$
a_{\mathrm{d}}=\mathrm{i}\left(k \cdot \nabla_{k}+\frac{3}{2}\right) \text {. }
$$

The formal relation $\Lambda=\mathrm{i}\left[L_{0}, A\right]$ follows from $\mathrm{i}\left[\omega, a_{\mathrm{d}}\right]=\omega$. The selfadjoint operator representing the quadratic form $\mathrm{i}\left[L_{\lambda, \theta}, A\right]$ is easily calculated to be (see (112))

$$
\begin{aligned}
C_{1}= & \Lambda+\lambda I_{1} \\
I_{1}= & G_{+} \otimes \mathbb{1}_{\mathbb{C}^{d}} \otimes\left\{a_{\mathcal{F}}\left(a_{\mathrm{d}} \sqrt{1+\rho} g\right) \otimes \mathbb{1}_{\mathcal{F}}-\mathbb{1}_{\mathcal{F}} \otimes a_{\mathcal{F}}^{*}\left(a_{\mathrm{d}} \sqrt{\rho} \bar{g}\right)\right\}+\operatorname{adj} . \\
& -\mathbb{1}_{\mathbb{C}^{d}} \otimes \mathcal{C}_{1} G_{+} \mathcal{C}_{1} \otimes\left\{a_{\mathcal{F}}^{*}\left(a_{\mathrm{d}} \sqrt{\rho} g\right) \otimes \mathbb{1}_{\mathcal{F}}-\mathbb{1}_{\mathcal{F}} \otimes a_{\mathcal{F}}\left(a_{\mathrm{d}} \sqrt{1+\rho} \bar{g}\right)\right\}+\text { adj. }
\end{aligned}
$$

Similar expressions are obtained for the higher commutators of $L_{\lambda, \theta}$ with $A$. Assumption (A1) guarantees that

$$
(1+1 / \sqrt{\omega})\left(a_{\mathrm{d}}\right)^{j} \sqrt{\rho} g \text { and }(1+1 / \sqrt{\omega})\left(a_{\mathrm{d}}\right)^{j} \sqrt{1+\rho} g
$$

are in $L^{2}\left(\mathbb{R}^{3}, \mathrm{~d}^{3} k\right)$, for $j=0, \ldots, 4$, so the commutators of $L_{\lambda, \theta}$ with $A$, up to order four, are represented by selfadjoint operators (satisfying the technical requirements needed in the proof of the virial theorem). 
Theorem 2 (Virial Theorem, regularity of eigenvectors of $L_{\lambda, \theta},[18]$ ). Assume condition (A1) and let $\theta \in[-\pi, \pi]$ be fixed. If $\psi$ is an eigenfunction of $L_{\lambda, \theta}$ then $\psi$ is in the form domain of $C_{1}$, (128), and

$$
\left\langle\psi, C_{1} \psi\right\rangle=0 .
$$

There is a constant $c$ which does not depend on $\theta \in[-\pi, \pi]$ nor on $\beta \geq \beta_{0}$, for any $\beta_{0}>0$ fixed, such that

$$
\left\|\Lambda^{1 / 2} \psi\right\| \leq c|\lambda|\|\psi\| .
$$

Remarks. 1) Relation (131) seems "obvious" from a formal point of view, writing $C_{1}=\mathrm{i}\left[L_{\lambda, \theta}, A\right]=\mathrm{i}\left[L_{\lambda, \theta}-e, A\right]$, and using that $\left(L_{\lambda, \theta}-e\right)^{*}=L_{\lambda, \theta}-e$, where $L_{\lambda, \theta} \psi=e \psi$. A proof of (131) is certainly not trivial, though, and considerable effort has been spent by many authors to establish "Virial Theorems" (see e.g. [1] and [13] for an overview, and [17, 9] for approaches similar to ours).

2) The regularity bound (132) follows easily from (131) and (128) and from the standard fact that $I_{1}$ is infinitesimally small relative to $\Lambda^{1 / 2}$ (Kato), so that $0=\left\langle\psi, C_{1} \psi\right\rangle \geq(1-\epsilon)\langle\psi, \Lambda \psi\rangle-\frac{\lambda^{2}}{\epsilon} c\|\psi\|^{2}$, for any $\epsilon>0$, for some constant $c$ independent of $\theta$ and $\beta$, as mentioned in the theorem. We refer for a more complete exposition of this to [9].

3) Theorem 2 is a special case of the Virial Theorem given in [18].

Our next result describes the structure of $\operatorname{Ker} L_{\lambda, \theta}$. Let $P(\Lambda \leq x)$ stand for the spectral projection of $\Lambda$ onto the interval $[0, x]$.

Theorem 3 (Structure of the kernel of $L_{\lambda, \theta},[\mathbf{1 8}]$ ). Assume Conditions (A1), (A2) and let $\theta \in[-\pi, \pi]$ be fixed. There is a number $\lambda_{0}>0$ s.t. if $0<|\lambda|<\lambda_{0}$ then any normalized $\psi_{\lambda} \in \operatorname{Ker}\left(L_{\lambda, \theta}\right)$ satisfies

$$
\psi_{\lambda}=\Omega_{1, \beta} \otimes\left(P(\Lambda \leq|\lambda|) \chi_{\lambda, \theta}\right)+O\left(\lambda^{0}\right),
$$

for some $\chi_{\lambda, \theta} \in \mathcal{F} \otimes \mathcal{F}$ satisfying $\left\|\chi_{\lambda, \theta}\right\| \geq 1-O\left(\lambda^{0}\right)$. Here and in (133), $O\left(\lambda^{0}\right)$ denotes a vector in $\mathcal{H}_{1} \otimes \mathcal{F} \otimes \mathcal{F}$ whose norm vanishes in the limit $\lambda \rightarrow 0$ (uniformly in $\theta \in[-\pi, \pi]$ and in $\beta \geq \beta_{0}$, for any $\beta_{0}>0$ fixed), and $\Omega_{1, \beta}$ is the Gibbs vector (63). The constant $\lambda_{0}$ does not depend on $\theta \in[-\pi, \pi]$, and it is uniform in $\beta \geq \beta_{0}$, for any fixed $\beta_{0}>0$.

The proof of this theorem, given in [18], relies on a positive commutator estimate and Theorem 2. Expansion (133) implies that the only vector in the kernel of $L_{\lambda, \theta}$ which does not converge weakly to zero, as $\lambda \rightarrow 0$, is the interacting KMS state $\Omega_{\beta, \lambda}^{\theta}$, (116). This information on the kernel of $L_{\lambda, \theta}$ alone enters our proof of Theorem 1, see Section 3.5.

Corollary 4 ([18]). Assume Conditions (A1) and (A2) and let $P_{\beta, \lambda}^{\theta}$ the projection onto the subspace spanned by the interacting KMS state $\Omega_{\beta, \lambda}^{\theta}$, 
(116). Let $\theta \in[-\pi, \pi]$ be fixed. Any normalized element $\psi_{\lambda} \in \operatorname{Ker}\left(L_{\lambda, \theta}\right) \cap$ $\left(\operatorname{Ran} P_{\beta, \lambda}^{\theta}\right)^{\perp}$ converges weakly to zero, as $\lambda \rightarrow 0$. The convergence is uniform in $\theta \in[-\pi, \pi]$ and in $\beta \geq \beta_{0}$, for any $\beta_{0}>0$ fixed.

\subsection{Discussion of Theorem 1 v.s. "return to equilibrium", and relation with infrared regularity of the coupling}

A central tool in our analysis of the time-asymptotic behaviour of the system is the virial theorem, whose use imposes regularity conditions on the interaction. In particular, we must be able to control the commutators of $L_{\lambda, \theta}$ with the conjugate operator $A$ of degree up to four (see previous section). Depending on the choice of $A$ this will impose more or less restrictive requirements on the interaction. A convenient choice for $A$ is obtained by representing $\mathcal{F} \otimes \mathcal{F}$ as $\mathcal{F}\left(L^{2}\left(\mathbb{R} \times S^{2}, \mathrm{~d} u \times \mathrm{d} \sigma\right)\right)$ and choosing $A=\mathrm{d} \Gamma\left(\mathrm{i} \partial_{u}\right)$ (translation generator). This choice, introduced in [14], has proven to be very useful and was adopted in $[17,6,9,12,10]$. The commutator of the non-interacting Liouvillian $L_{0}=\mathrm{d} \Gamma(u)$ with $A$ (multiplied by $i$ ) is just $N=\mathrm{d} \Gamma(\mathbb{1})$, the number operator in $\mathcal{F}\left(L^{2}\left(\mathbb{R} \times S^{2}, \mathrm{~d} u \times \mathrm{d} \sigma\right)\right)$, which has a one-dimensional kernel and a spectral gap at zero. We may explain the usefulness of the gap as follows. If one carries out the proof of Theorem 3 with the translation generator as the conjugate operator then the role of $\Lambda,(125)$, is taken by $N$, and relation (133) is replaced by $\left\|P_{1, \beta} P(N \leq|\lambda|) \psi_{\lambda}\right\|=1-O\left(\lambda^{0}\right)$, where $P_{1, \beta}=\left|\Omega_{1, \beta}\right\rangle\left\langle\Omega_{1, \beta}\right|$. But for $|\lambda|<1, P_{\beta, 1} P(N \leq|\lambda|)$ is just the projection $\left|\Omega_{\beta, 0}\right\rangle\left\langle\Omega_{\beta, 0}\right|$ onto the span of the non-interacting KMS state, so one has $\left|\left\langle\Omega_{\beta, 0}, \psi_{\lambda}\right\rangle\right|=1-O\left(\lambda^{0}\right)$. Since $\Omega_{\beta, 0}$ is close to $\Omega_{\beta, \lambda}^{\theta}$ for small values of $\lambda$, this means that there are no elements in the kernel of $L_{\lambda, \theta}$ which are orthogonal to $\Omega_{\beta, \lambda}^{\theta}$, provided $|\lambda|$ is small enough. Hence the kernel of $L_{\lambda, \theta}$ has dimension one. A consequence of the simplicity of $\operatorname{Ker} L_{\lambda, \theta}$ is that return to equilibrium holds, as explained in Subsection 1.5 .

The disadvantage of the translation generator is that its use requires (too) restricitve infrared regularity on the form factor. Indeed, the $j$-th commutator of the interaction with the translation generator involves the $j$-th derivative of the fuction $\frac{g}{\sqrt{e^{\beta \omega}-1}}$, so an infrared singular behaviour of this function is worsened by each application of the commutator (and we require those derivatives to be square integrable!). As a result, the case $g(0) \neq 0$ cannot be treated.

The remedy is to develop the theory with a conjugate operator $A$ which does not affect the infrared behaviour of $\frac{g}{\sqrt{e^{\beta \omega}-1}}$ in a negative way. The operator (126) (dilation generator) is a good candidate (one could as well take operators interpolating between the translation and the dilation generator). The disadvantage of using the dilation generator is that its commutator with the non-interacting Liouvillian gives the operator $\Lambda$, which still has a onedimensional kernel, but does not have a spectral gap at zero. This means that 
we cannot show that the kernel of $L_{\lambda, \theta}$ is simple, but we only have expansion (133).

\subsection{Outline of the proof of Theorem 1}

It is enough to show that for fixed $\theta \in S^{1}$,

$$
\begin{aligned}
\lim _{\lambda \rightarrow 0} \lim _{T \rightarrow \infty} \frac{1}{T} \int_{0}^{T}\left\langle\Omega_{\beta, \lambda}^{\theta}, B_{\theta}^{*} \sigma_{\lambda, \theta}^{t}\left(A_{\theta}\right) B_{\theta} \Omega_{\beta, \lambda}^{\theta}\right\rangle \mathrm{d} t \\
=\left\langle\Omega_{\beta, 0}^{\theta}, B_{\theta}^{*} B_{\theta} \Omega_{\beta, 0}^{\theta}\right\rangle\left\langle\Omega_{\beta, 0}^{\theta}, A_{\theta} \Omega_{\beta, 0}^{\theta}\right\rangle .
\end{aligned}
$$

As in Subsection 1.5 we use the KMS condition and the fact that $\Omega_{\beta, \lambda}^{\theta} \in$ $\operatorname{Ker} L_{\lambda, \theta}$ to obtain

$$
\left\langle\Omega_{\beta, \lambda}^{\theta}, B_{\theta}^{*} \sigma_{\lambda, \theta}^{t}\left(A_{\theta}\right) B_{\theta} \Omega_{\beta, \lambda}^{\theta}\right\rangle=\left\langle\Omega_{\beta, \lambda}^{\theta}, \sigma_{\lambda, \theta}^{-\mathrm{i} \beta}\left(B_{\theta}\right) B_{\theta}^{*} \mathrm{e}^{\mathrm{i} t L_{\lambda, \theta}} A_{\theta} \Omega_{\beta, \lambda}^{\theta}\right\rangle .
$$

A word of caution is in place here. The expression $\sigma_{\lambda, \theta}^{-\mathrm{i} \beta}\left(B_{\theta}\right)$ is only defined for "nice" (analytic) observables $B$. One can perform the proof first for such $B$ and then use a density argument to conclude that the result holds for all $B$. (In [18] an alternative route is taken, using the fact that $\Omega_{\beta, \lambda}^{\theta}$ is cyclic for the commutant $\left(\mathfrak{M}_{1} \otimes \mathfrak{M}_{0}\right)^{\prime}$.)

Let $\left\{\psi_{j, \lambda}^{\theta}\right\}_{j} \cup\left\{\Omega_{\beta, \lambda}^{\theta}\right\}$ be an orthonormal basis of $\operatorname{Ker} L_{\lambda, \theta}$. The von Neumann ergodic theorem gives

$$
\begin{aligned}
\lim _{T \rightarrow \infty} \frac{1}{T} \int_{0}^{T}(135) \mathrm{d} t= & \left\langle\Omega_{\beta, \lambda}^{\theta}, \sigma_{\lambda, \theta}^{-\mathrm{i} \beta}\left(B_{\theta}\right) B_{\theta}^{*} \Omega_{\beta, \lambda}^{\theta}\right\rangle\left\langle\Omega_{\beta, \lambda}^{\theta}, A_{\theta} \Omega_{\beta, \lambda}^{\theta}\right\rangle \\
& +\sum_{j}\left\langle\Omega_{\beta, \lambda}^{\theta}, \sigma_{\lambda, \theta}^{-\mathrm{i} \beta}\left(B_{\theta}\right) B_{\theta}^{*} \psi_{j, \lambda}^{\theta}\right\rangle\left\langle\psi_{j, \lambda}^{\theta}, A_{\theta} \Omega_{\beta, \lambda}^{\theta}\right\rangle
\end{aligned}
$$

In the limit $\lambda \rightarrow 0$ the sum on the r.h.s. of (136) disappears, due to Corollary 4 .

\section{References}

1. W. Amrein, A. Boutet de Monvel, V. Georgescu: $C_{0}$-Groups, Commutator Methods and Spectral Theory of $N$-body Hamiltonians. Basel-Boston-Berlin: Birkhäuser, 1996

2. H. Araki, E. Woods: J. Math. Phys. 4, 637 (1963)

3. V. Bach, J. Fröhlich, I.M. Sigal, I.M.: J. Math. Phys. 41, no. 6, 3985 (2000)

4. Bratteli, O., Robinson, D.W., Operator Algebras and Quantum Statistical Mechanics I, II. Texts and Monographs in Physics, Springer-Verlag, 1987

5. J.T. Cannon: Commun. Math. Phys., 29, 89 (1973)

6. J. Dereziński, J., V. Jakšić: Ann. Henri Poincaré 4, no. 4, 739 (2003)

7. J. Dereziński, V. Jakšić, C.-A. Pillet: Rev. Math. Phys. 15, no. 5, 447 (2003) 
8. R.B. Diener, B. Wu, M.G. Raizen, Q. Niu: http://xxx.lanl.gov/abs/condmat/0201159 (2003)

9. J. Fröhlich, M. Merkli: Math. Phys. Anal. Geom. 7, no.3, 239 (2004)

10. J. Fröhlich, M. Merkli: Commun. Math. Phys. 251, no. 2, 235 (2004)

11. J. Fröhlich, M. Merkli, D. Ueltschi: Ann. Henri Poincaré 4 no. 5, 897 (2003)

12. J. Fröhlich, M. Merkli, I.M. Sigal: J. Statist. Phys. 116, no. 1-4, 311 (2004)

13. V. Georgescu, C. Gérard: Commun. Math. Phys. 208275 (1999)

14. V. Jakšić, C.-A. Pillet: Commun. Math. Phys. 176, 619 (1996)

15. V. Jakšić, C.-A. Pillet: Commun. Math. Phys. 178, 627 (1996)

16. J.T. Lewis, J.V. Pulè: Commun. Math. Phys. 36, 1 (1974)

17. M. Merkli: Commun. Math. Phys. 223, 327 (2001)

18. M. Merkli: to appear in Commun. Math. Phys. (2005)

19. M. Merkli: The Ideal Quantum Gas. Lecture notes for the summerschool on open quantum systems, Institut Fourier, Grenoble, 2003 